\title{
Wrinkle Patterns of Anisotropic Crystal Films on Viscoelastic Substrates
}

\author{
Se Hyuk Im and Rui Huang* \\ Department of Aerospace Engineering and Engineering Mechanics \\ University of Texas, Austin, TX 78712
}

\begin{abstract}
This paper presents a nonlinear mathematical model for evolution of wrinkle patterns of an anisotropic crystal film on a viscoelastic substrate layer. The underlying mechanism of wrinkling has been generally understood as a stress-driven instability. Previously, theoretical studies on wrinkling have assumed isotropic elastic properties for the film. Motivated by recent experimental observations of ordered wrinkle patterns in single-crystal thin films, this paper develops a theoretical model coupling anisotropic elastic deformation of a crystal film with viscoelastic deformation of a thin substrate layer. A linear perturbation analysis is performed to predict onset of the wrinkling instability and the initial evolution kinetics; an energy minimization method is adopted to analyze wrinkle patterns at the equilibrium states. For a cubic crystal film under an equi-biaxial compression, orthogonally ordered wrinkle patterns are predicted at both the initial stage and the equilibrium state. This is confirmed by numerical simulations of evolving wrinkle patterns. By varying the residual stresses in the film, numerical simulations show that a variety of wrinkle patterns (e.g., orthogonal, parallel, zigzag, and checkerboard patterns) emerge as a result of the competition between the material anisotropy and the stress anisotropy.
\end{abstract}

Keywords: anisotropic material; pattern evolution; thin film; instability

\footnotetext{
* Corresponding author. Tel: +1-512-471-7558; Fax: +1-512-471-5500; Email: ruihuang@mail.utexas.edu.
} 


\section{Introduction}

Complex wrinkle patterns have been observed in various thin-film systems with integrated hard and soft materials (e.g., Tolpygo and Clarke, 1998; Bowden et al., 1998; Hobart et al., 2000; Volynskii et al., 2000; Yoo and Lee, 2003). For many applications of the integrated material structures, wrinkling is undesirable as it may lead to failure by delamination or fracture (e.g., Suo, 1995; Martin et al., 2000; Mumm et al., 2001; Yin et al., 2003; Peterson, 2006). On the other hand, wrinkling has also been exploited as an enabling mechanism for a variety of applications, such as stretchable electronics (Watanabe et al., 2002; Lacour et al., 2003; Choi et al., 2007), micro/nanoscale surface patterning (Huck et al., 2000; Serrano and Cahill, 2002; Ohzono and Shimomura, 2004; Chan and Crosby, 2006), optical phase grating (Harrison et al., 2004), microfluidic sieves (Efimenko et al., 2005), smart adhesion (Chan et al., 2008), and metrology aid for measuring mechanical properties of thin films (Stafford et al, 2004). A collection of physical understanding and applications of the wrinkling phenomena was recently reviewed by Genzer and Groenewold (2006).

Theoretical studies of surface wrinkling may be traced back to 1940s when wrinkling of face struts was analyzed as a form of local elastic instability in structural sandwich panels (e.g., Gough et al., 1940; Wan, 1947; Goodier and Neou, 1951); an account of the historical development was well documented by Allen (1969). Later, a series of works by Biot extended the wrinkling theory to viscoelastic layers (Biot, 1957) and rubber-like nonlinear elastic media under finite strain (Biot, 1963 and 1965). The early studies of wrinkling focused on the critical conditions for the onset of instability. Recent advances in micro/nanoscale fabrication and measurements have renewed the interest in mechanics of wrinkling beyond the initial instability. An energy-based approach has been widely adopted for post-instability analysis to determine the 
equilibrium states of wrinkling for an elastic film on an elastic substrate (Groenewold, 2001; Chen and Hutchinson, 2004; Huang et al., 2005; Jiang et al., 2007; Song et al., 2008). Temporal evolution of wrinkles has also been analyzed for an elastic film on a viscous or viscoelastic substrates (Sridhar et al., 2001; Huang and Suo, 2002a and 2002b; Huang, 2005; Im and Huang, 2005; Huang and Im, 2006).

Most of the previous studies have assumed isotropic, linear elastic properties for the film, while the substrate may be linear elastic, hyperelastic, or linearly viscoelastic (including viscous Newtonian fluids). Under an equi-biaxial compressive stress, the wrinkle pattern of an isotropic elastic film characteristically exhibits the lack of ordering such as the labyrinth pattern commonly observed in experiments, although some ordered wrinkle patterns (e.g., parallel stripes, herringbone or zigzag, and checkerboard) have been theoretically predicted and occasionally observed under particular stress and boundary conditions. The rotational symmetry in the isotropic system is believed to be responsible for the disordered wrinkle patterns. The symmetry can be broken either by invoking an anisotropic biaxial stress or in a layered material system with anisotropic mechanical properties. For example, Hobart et al. (2000) observed wrinkling of single-crystal silicon-germanium (SiGe) alloy films on a glass layer annealed at an elevated temperature, where the SiGe is a cubic crystal with anisotropic elastic properties. More detailed experiments (Peterson, 2006; Peterson et al., 2006) showed that the SiGe film preferentially wrinkles in two orthogonal directions, aligned with the $<100>$ axes of the cubic crystal. Similar orthogonal patterns were observed by Yu et al. (2005) for a SiGe/oxide film stack. Recently, Choi et al. (2007) observed zigzag wrinkle patterns of single-crystal Si films bonded to prestrained polydimethylsiloxane (PDMS) substrate, where the jog angle of the zigzag was close to $90^{\circ}$, although no alignment with the crystal axes was reported. Motivated by these 
experimental observations, this paper develops a theoretical model for wrinkling of an anisotropic elastic film on a thin viscoelastic substrate layer. Such a model allows us to analyze the wrinkle patterns and their evolution under various stress states. In particular, the effects of anisotropic elastic property of a cubic crystal film on wrinkle patterns at both the initial stage and the equilibrium state are elucidated in the present study by analytical solutions and numerical simulations.

The remainder of this paper is organized as follows. Section 2 presents the model formulation, coupling a nonlinear plate theory for the anisotropic elastic film with a thin-layer model for the viscoelastic substrate. Section 3 performs a linear perturbation analysis, from which the critical condition and initial evolution kinetics of wrinkling are predicted. Section 4 develops analytical solutions for equilibrium wrinkle states by a nonlinear energy minimization approach. In Section 5, numerical simulations are conducted for both isotropic and anisotropic elastic films, showing the evolution and transition of wrinkle patterns under various stress conditions. Section 6 concludes with a summary of the results.

\section{Model Formulation}

Figure 1 illustrates the model structure considered in this study: an anisotropic elastic film of thickness $h$ lying on a viscoelastic substrate layer of thickness $H$, which in turn lies on a rigid foundation. The material system has a reference state (Fig. 1a), when both layers are flat and the elastic film is subject to a uniformly distributed residual stress. The substrate layer is assumed to be stress free at the reference state. Figure 1b sketches a wrinkled state, where the elastic film undergoes a buckling deformation and the viscoelastic layer deforms concomitantly. The interface between the layers is assumed to remain bonded. 
To be specific, we consider a cubic crystal film with the surface normal in the [001] crystal direction. For convenience, a Cartesian coordinate system is set up at the reference state such that the $x-y$ plane parallels to the film surface and the in-plane axes align with the [100] and [010] directions of the crystal (see Fig. 1a). In general, the residual stress in the film has three inplane components, $\sigma_{x x}^{R}, \sigma_{y y}^{R}$, and $\sigma_{x y}^{R}$, as illustrated in Figure 2a. The stress state can also be represented by two principal stresses ( $\sigma_{1}$ and $\sigma_{2}$ ) and the corresponding principal angle $\left(\theta_{p}\right)$, as illustrated in Figure 2b. The tensor property of the stress gives the principal stresses and the principal angle in terms of the original stress components, namely,

$$
\begin{aligned}
& \sigma_{1,2}=\frac{\sigma_{x x}^{R}+\sigma_{y y}^{R}}{2} \pm \sqrt{\left(\frac{\sigma_{x x}^{R}-\sigma_{y y}^{R}}{2}\right)^{2}+\left(\sigma_{x y}^{R}\right)^{2}}, \\
& \tan 2 \theta_{p}=\frac{2 \sigma_{x y}^{R}}{\sigma_{x x}^{R}-\sigma_{y y}^{R}} .
\end{aligned}
$$

As a necessary condition for the film to wrinkle, at least one of the two principal stresses must be negative (compressive). As will be shown later, the wrinkle pattern of the anisotropic film depends on the ratio between the two principal stresses and the principal angle. Typically, the residual stress is equi-biaxial $\left(\sigma_{1}=\sigma_{2}\right)$ in an infinite blanket film and nearly uniaxial $\left(\sigma_{2}=0\right)$ in a narrow strip. In a film of finite dimension, the residual stress state varies from equi-biaxial in the center region to uniaxial near the free edges. A non-uniformly distributed residual stress may lead to interesting wrinkle patterns (see an example in Huang et al., 2005); however, the present study considers only uniform residual stresses at the reference state. 


\subsection{Anisotropic elastic deformation of a crystal film}

Under the condition of small deformation, the stress and strain in a crystal are related by the generalized Hooke’s law of linear elasticity:

$$
\sigma_{i j}=C_{i j k l} \varepsilon_{k l}
$$

where $C_{i j k l}$ is the elastic constants and the subscripts, $i, j, k, l$, take values from 1 to 3 for the Cartesian coordinates. Equation (3) can be written in a matrix form using the abbreviated notation $C_{p q}$ for the elastic constants, with the subscripts $p$ and $q$ taking values from 1 to 6 . In particular, for an orthotropic material, we have

$$
\left[\begin{array}{c}
\sigma_{x x} \\
\sigma_{y y} \\
\sigma_{z z} \\
\sigma_{y z} \\
\sigma_{z x} \\
\sigma_{x y}
\end{array}\right]=\left[\begin{array}{cccccc}
C_{11} & C_{12} & C_{13} & 0 & 0 & 0 \\
C_{12} & C_{22} & C_{23} & 0 & 0 & 0 \\
C_{13} & C_{23} & C_{33} & 0 & 0 & 0 \\
0 & 0 & 0 & 2 C_{44} & 0 & 0 \\
0 & 0 & 0 & 0 & 2 C_{55} & 0 \\
0 & 0 & 0 & 0 & 0 & 2 C_{66}
\end{array}\right]\left[\begin{array}{c}
\varepsilon_{x x} \\
\varepsilon_{y y} \\
\varepsilon_{z z} \\
\varepsilon_{y z} \\
\varepsilon_{z x} \\
\varepsilon_{x y}
\end{array}\right] .
$$

For a cubic crystal, the stress-strain relation takes the same form as Eq. (4), but with $C_{11}=C_{22}=C_{33}, C_{44}=C_{55}=C_{66}$, and $C_{12}=C_{23}=C_{13}$. Additional symmetry for an isotropic material leads to the relation, $C_{11}-C_{12}=2 C_{66}$, reducing the number of independent elastic constants to two.

In the present study, the crystal film is modeled as a thin elastic plate. Following the classical plate theory (Landau and Lifshitz, 1959; Timoshenko and Woinowsky-Krieger, 1987), we set $\sigma_{z x}=\sigma_{z y}=\sigma_{z z}=0$ in Eq. (4) and obtain the in-plane stress-strain relation: 


$$
\left[\begin{array}{l}
\sigma_{x x} \\
\sigma_{y y} \\
\sigma_{x y}
\end{array}\right]=\left[\begin{array}{ccc}
\bar{C}_{11} & \bar{C}_{12} & 0 \\
\bar{C}_{12} & \bar{C}_{22} & 0 \\
0 & 0 & 2 C_{66}
\end{array}\right]\left[\begin{array}{c}
\varepsilon_{x x} \\
\varepsilon_{y y} \\
\varepsilon_{x y}
\end{array}\right],
$$

where $\bar{C}_{\alpha \beta}=C_{\alpha \beta}-C_{3 \alpha} C_{3 \beta} / C_{33}$ for $\alpha, \beta=1$ or 2 . For a cubic crystal plate, $\bar{C}_{11}=\bar{C}_{22}=C_{11}-C_{12}^{2} / C_{11}$, and $\bar{C}_{12}=C_{12}-C_{12}^{2} / C_{11}$. For an isotropic elastic plate, the reduced elastic constants can be expressed in terms of Young's modulus $(E)$ and Poisson's ratio $(v)$, namely, $\bar{C}_{11}=\bar{C}_{22}=E /\left(1-v^{2}\right), \bar{C}_{12}=v E /\left(1-v^{2}\right)$, and $C_{66}=E /[2(1+v)]$.

Let $u_{x}$ and $u_{y}$ be the in-plane displacements and $w$ the lateral deflection of the film. Upon wrinkling, the membrane strain components are

$$
\begin{aligned}
& \varepsilon_{x x}=\frac{\partial u_{x}}{\partial x}+\frac{1}{2}\left(\frac{\partial w}{\partial x}\right)^{2}, \\
& \varepsilon_{y y}=\frac{\partial u_{y}}{\partial y}+\frac{1}{2}\left(\frac{\partial w}{\partial y}\right)^{2}, \\
& \varepsilon_{x y}=\frac{1}{2}\left(\frac{\partial u_{x}}{\partial y}+\frac{\partial u_{y}}{\partial x}\right)+\frac{1}{2} \frac{\partial w}{\partial x} \frac{\partial w}{\partial y} .
\end{aligned}
$$

A nonlinear term is included in each strain component to account for the geometrical nonlinearity due to moderately large deflection of the elastic film, same as the nonlinear vonKarman equations for an isotropic elastic plate. It may be noted that the displacements are measured relative to the reference state with the residual stress $\sigma_{i j}^{R}$, and thus the strain in Eq. (6) describes an incremental deformation from the stressed reference state. As long as the total deformation of the film is sufficiently small for the material to behave in the linearly elastic regime, the total stress in the film is approximately $\sigma_{i j}^{R}+\sigma_{i j}$, where $\sigma_{i j}$ is related to the incremental strain by Eq. (5) (Biot, 1965). 
The interaction between the film and the substrate layer exerts normal and shear tractions onto the lower surface of the film. Similar to the isotropic plate theory, equilibrium of the anisotropic elastic film requires that

$$
\begin{gathered}
\frac{h^{3}}{12}\left[\bar{C}_{11} \frac{\partial^{4} w}{\partial x^{4}}+2\left(\bar{C}_{12}+2 C_{66}\right) \frac{\partial^{4} w}{\partial x^{2} \partial y^{2}}+\bar{C}_{22} \frac{\partial^{4} w}{\partial y^{4}}\right] \\
-h\left[\frac{\partial}{\partial x}\left(\left(\sigma_{x x}^{R}+\sigma_{x x}\right) \frac{\partial w}{\partial x}+\left(\sigma_{x y}^{R}+\sigma_{x y}\right) \frac{\partial w}{\partial y}\right)+\frac{\partial}{\partial y}\left(\left(\sigma_{y y}^{R}+\sigma_{y y}\right) \frac{\partial w}{\partial y}+\left(\sigma_{x y}^{R}+\sigma_{x y}\right) \frac{\partial w}{\partial x}\right)\right]=p \\
\frac{\partial \sigma_{x x}}{\partial x}+\frac{\partial \sigma_{x y}}{\partial y}=\frac{T_{x}}{h} \\
\frac{\partial \sigma_{x y}}{\partial x}+\frac{\partial \sigma_{y y}}{\partial y}=\frac{T_{y}}{h}
\end{gathered}
$$

where $p$ is the pressure (negative normal traction), $T_{x}$ and $T_{y}$ are the shear tractions. Similar equations for orthotropic thin plates have been developed previously (Bloom and Coffin, 2000).

\subsection{Viscoelastic evolution equations}

With the assumption of perfectly bonded interface between the film and the substrate, the same tractions ( $p, T_{x}$ and $T_{y}$ ) act on the upper surface of the substrate layer. In response, the viscoelastic layer deforms in a time-dependent manner. Based on the linear theory of viscoelasticity and the elasticity-viscoelasticity correspondence principle (Christensen, 1982), we previously derived a thin-layer approximation of the viscoelastic deformation (Im and Huang, 2005). The time-dependent relaxation shear modulus of the viscoelastic layer is taken to be Kelvin type, with a rubbery modulus $\mu_{R}$ and a viscosity $\eta$, while the Poisson's ratio ( $\left.v \neq 0.5\right)$ is assumed to be independent of time. The displacement rates at the upper surface of the viscoelastic layer are then related to the tractions as follows: 


$$
\begin{aligned}
& \frac{\partial w}{\partial t}=-\frac{1-2 v}{2(1-v)} \frac{H}{\eta} p-\frac{\mu_{R}}{\eta} w, \\
& \frac{\partial u_{x}}{\partial t}=\frac{H}{\eta} T_{x}-\frac{\mu_{R}}{\eta} u_{x}, \\
& \frac{\partial u_{y}}{\partial t}=\frac{H}{\eta} T_{y}-\frac{\mu_{R}}{\eta} u_{y} .
\end{aligned}
$$

We have assumed that both the displacements and the tractions are continuous across the interface between the film and the substrate layer. We have also assumed that the evolution process is sufficiently slow such that the inertia effect is negligible and the elastic film remains in equilibrium during evolution. Consequently, the equilibrium equations of the elastic film in Eqs. (7)-(9) are coupled with the viscoelastic evolution equations, forming a complete temporalspatial system.

Despite the limitations in the viscoelastic property and the layer thickness, the present model captures essential features of the viscoelastic wrinkle evolution, such as the kinetics of wrinkle growth and the equilibrium states at the long-time limit (Huang and Im, 2006). Extension to more general cases is possible, but not pursued in the present study. Notably, Biot (1957) developed a general instability theory for a viscoelastic layer on a semi-infinite viscoelastic substrate or embedded in an infinite viscoelastic medium, which may be extended for three-dimensional post-instability analysis. Alternatively, a Fourier transform method similar to that by Huang et al. (2005) can be employed for an elastic film on an infinitely thick viscoelastic substrate. For viscoelastic substrates of finite thickness, only two-dimensional analysis is available (Huang, 2005) to the best of our knowledge. 


\section{Linear Perturbation Analysis}

An arbitrary wrinkle pattern can be considered as a superposition of many Fourier components, each of which is designated by a wave number $k$ (or equivalently, wavelength $\lambda=2 \pi / k)$, an angle of the wave vector $\theta$, and an amplitude $A$. The amplitude is a function of time as the wrinkle evolves. For the linear analysis, we may consider evoltuion of each individual Fourier component as a small perurbation to the reference state. In particular, the lateral deflection of the film takes the form

$$
w(x, y, t)=A(t) \cos [k(x \cos \theta+y \sin \theta)] .
$$

Here, the angle $\theta$ is measured from the $x$-axis or the [100] axis for the cubic crystal film.

Linearization of Eqs. (7-9) uncouples the in-plane displacements from the lateral deflection. Thus, only Eq. (10) needs to be solved at the linear regime. By inserting Eq. (13) into Eq. (7) and keeping only the linear terms, we obtain the pressure at the interface:

$$
p=\left[\frac{1}{12} \bar{E}_{\theta}(k h)^{4}+\sigma_{\theta}(k h)^{2}\right] \frac{A(t)}{h} \cos [k(x \cos \theta+y \sin \theta)],
$$

where

$$
\begin{aligned}
& \bar{E}_{\theta}=\bar{C}_{11} \cos ^{4} \theta+\bar{C}_{22} \sin ^{4} \theta+2\left(\bar{C}_{12}+2 C_{66}\right) \sin ^{2} \theta \cos ^{2} \theta, \\
& \sigma_{\theta}=\sigma_{x x}^{R} \cos ^{2} \theta+\sigma_{y y}^{R} \sin ^{2} \theta+2 \sigma_{x y}^{R} \sin \theta \cos \theta .
\end{aligned}
$$

Substituting Eqs. (13) and (14) into Eq. (10), we obtain that

$$
\frac{d A}{d t}=\frac{\alpha_{\theta}-\mu_{R}}{\eta} A(t)
$$

where

$$
\alpha_{\theta}=\frac{(1-2 v) H}{24(1-v) h}\left[-\bar{E}_{\theta}(k h)^{4}-12 \sigma_{\theta}(k h)^{2}\right]
$$

Solving Eq. (17) leads to 


$$
A(t)=A_{0} \exp \left(s_{\theta} \frac{t}{\tau}\right)
$$

where $A_{0}$ is a constant for the initial amplitude, $\tau=\eta / C_{11}$ is a characteristic time scale, and $s_{\theta}=\left(\alpha_{\theta}-\mu_{R}\right) / C_{11}$ is the dimensionless growth rate of the perturbation amplitude.

The result from the linear analysis is identical to that for an isotropic elastic film (Im and Huang, 2005) except for the dependence of the growth rate on the angle $\theta$ through $\bar{E}_{\theta}$ and $\sigma_{\theta}$. As defined in Eq. (16), the stress $\sigma_{\theta}$ is simply the normal component of the residual stress acting on a rotated section with the angle $\theta$, as illustrated in Fig. 2c. When the residual stress at the reference state is equi-biaxial, i.e., $\sigma_{x x}^{R}=\sigma_{y y}^{R}=\sigma_{1}$ and $\sigma_{x y}^{R}=0$, we have $\sigma_{\theta}=\sigma_{1}$, independent of the angle $\theta$. Thus, an equi-bixial stress is isotropic. Otherwise, the stress state is anisotropic. In terms of the principal stresses, we rewrite $\sigma_{\theta}$ in form of

$$
\sigma_{\theta}=\sigma_{1}\left[1-\left(1-\frac{\sigma_{2}}{\sigma_{1}}\right) \sin ^{2}\left(\theta-\theta_{p}\right)\right] .
$$

Hence the ratio between the two principle stresses determines the angle dependence of $\sigma_{\theta}$ and represents the stress anisotropy.

The modulus $\bar{E}_{\theta}$ defined in Eq. (15) is essentially the plane-strain modulus in the direction of the wrinkle wave vector. For a cubic crystal film, $\bar{E}_{\theta}$ can be rewritten as

$$
\bar{E}_{\theta}=C_{11}\left[1-\left(\frac{C_{12}}{C_{11}}\right)^{2}\right]\left[1+\frac{(\xi-1) C_{11}}{2\left(C_{11}+C_{12}\right)} \sin ^{2} 2 \theta\right],
$$

where $\xi=2 C_{66} /\left(C_{11}-C_{12}\right)$ is the degree of elastic anisotropy. In the case of an isotropic material, $\xi=1$ and $\bar{E}_{\theta}$ reduces to $E /\left(1-v^{2}\right)$, independent of the angle. Table 1 lists the elastic constants and the degrees of elastic anisotropy for single-crystal $\mathrm{Si}$, Ge, and an alloy $\mathrm{Si}_{0.7} \mathrm{Ge}_{0.3}$. 
The elastic properties of the $\mathrm{Si}_{1-x} \mathrm{Ge}_{x}$ alloy $(0 \leq x \leq 1)$ are obtained by linear interpolation between those of Si and Ge with $x$ being the Ge content (Fitzgerold, 1995). From Eq. (21) it is noted that, when $\xi>1$, the plane-strain modulus of a cubic crystal maximizes at $<110>$ directions $\left(\theta= \pm 45^{\circ}\right)$ and minimizes at $<100>$ directions $\left(\theta=0\right.$ and $\left.90^{\circ}\right)$. The trend is opposite when $\xi<1$ (e.g., for single-crystal $\mathrm{Cr}, \mathrm{Mo}, \mathrm{NaCl}$, and $\mathrm{TiC}$ ).

For any particular angle $\theta$, the growth rate $s_{\theta}$ has a peak at the wavelength

$$
\lambda_{\theta m}=\pi h \sqrt{-\frac{2 \bar{E}_{\theta}}{3 \sigma_{\theta}}}
$$

The corresponding peak growth rate is zero at a critical stress

$$
\sigma_{\theta c}=-\sqrt{\frac{2}{3} \frac{1-v}{1-2 v} \frac{h}{H} \mu_{R} \bar{E}_{\theta}} .
$$

When the residual stress $-\sigma_{\theta}<-\sigma_{\theta c}$, the peak growth rate is negative and the crystal film at the reference state is stable against perturbations with the angle $\theta$. Otherwise, when $-\sigma_{\theta}>-\sigma_{\theta c}$, the peak growth rate is positive and the crystal film becomes unstable.

Both the wavelength $\lambda_{\theta m}$ and the critical stress $\sigma_{\theta c}$ vary with respect to the direction of the wrinkle wave vector for an anisotropic elastic film. Figure 3a plots the magnitude of the critical stress as a function of the angle $\theta$ for single-crystal $\mathrm{Si}$, Ge, and $\mathrm{Si}_{0.7} \mathrm{Ge}_{0.3}$ films, with $\mu_{R}=1.5 \mathrm{MPa}, v=0.45$, and $H / h=10$ for the substrate layer. The material anisotropy dictates the dependence of the critical stress on the wrinkle orientation. For the SiGe film, the critical stress is the lowest for wrinkling in the $<100>$ directions $\left(\theta=0\right.$ and $90^{\circ}$ ), and the highest for wrinkling in the $<110>$ directions $\left(\theta= \pm 45^{\circ}\right)$. The difference is roughly $10 \%$. Under an equibiaxial stress, the stability of the film is controlled by the lowest critical stress $\left(\sigma_{c}^{<100>}\right)$. Under a 
uniaxial stress, however, the critical stress depends on the direction of the stress with respect to the crystal axes. The critical stress strongly depends on the rubbery modulus of the substrate. Figure $3 \mathrm{~b}$ plots the minimum and maximum critical stresses for the $\mathrm{Si}_{0.7} \mathrm{Ge}_{0.3}$ film versus the rubbery modulus $\mu_{R}$. The critical stresses increase dramatically as the rubbery modulus exceeds 100 MPa. Plastic deformation of the crystal film by dislocation mechanisms (Matthews and Blakeslee, 1974) should be considered under high stress levels.

According to Equation (19), the wrinkle amplitude grows exponentially with time at the initial stage. Thus, the wavelength with the peak growth rate, $\lambda_{\theta m}$, dominates the initial wrinkle evolution. It is noted that $\lambda_{\theta m}$ depends on the stress $\sigma_{\theta}$, but independent of the rubbery modulus of the viscoelastic substrate. For a $30 \mathrm{~nm} \mathrm{Si}_{0.7} \mathrm{Ge}_{0.3}$ film on a $235 \mathrm{~nm}$ BPSG substrate, Peterson et al. (2006) measured the wrinkle wavelengths to be $0.91 \mu \mathrm{m}$ and $0.94 \mu \mathrm{m}$ under equi-biaxial and uniaxial stress states, respectively. The corresponding wavelengths predicted by Eq. (22) are 0.62 $\mu \mathrm{m}$ and $0.73 \mu \mathrm{m}$, with the stresses calculated from Eq. (5) with the mismatch strain -0.012 . The difference between the predicted and measured wavelengths may come from two sources: (1) Both experiments and theory have shown that the wrinkle wavelength coarsens over time (Yoo and Lee, 2003; Huang and Im, 2006). While Eq. (22) predicts the dominant wavelength at the early stage of wrinkle evolution, the measured values may have already undergone coarsening beyond the linear regime; (2) Even for the linear analysis, the thin-layer approximation of the viscoelastic substrate tends to underestimate the wrinkle wavelength, as shown previously for wrinkling of isotropic elastic films (Huang and Suo, 2002b; Huang 2005).

Figure 4 compares the spectra of the initial wrinkle growth rate for both isotropic and anisotropic elastic films under various residual stresses. Contours of the normalized growth rate $s_{\theta}$ are plotted in the plane spanning the $x$ and $y$ components of the wave vector, $k_{x}=k \cos \theta$ and 
$k_{y}=k \sin \theta$, both normalized by the film thickness $h$; only positive growth rates are plotted. In all the calculations, we set $\mu_{R} / C_{11}=10^{-5}, H / h=10$, and $v=0.45$. The major principal stress is fixed to be $\sigma_{1} / C_{11}=-0.003$, while the ratio $\sigma_{2} / \sigma_{1}$ is varied from 1 for equi-biaxial to 0 for uniaxial stress state. For the isotropic film, the growth spectrum is solely controlled by the stress ratio. Under an equi-biaxial residual stress $\left(\sigma_{2} / \sigma_{1}=1\right)$, the growth spectrum is isotropic as the wave vectors of all the growing modes reside in a circular ring region with no favored directions. When the two principal stresses differ, the rotational symmetry is broken and the growth rate peaks at a particular wave vector in the direction of the major principal stress. Therefore, the kinetically dominant wrinkle pattern at the initial stage changes from non-directional (e.g., the labyrinth pattern) to uni-directional (e.g., the parallel striped pattern). Similar symmetry breaking occurs in many other pattern evolution systems (e.g., Lu and Suo, 2002; Pang and Huang, 2007).

For the cubic crystal film ( $\mathrm{Si}_{0.7} \mathrm{Ge}_{0.3}$, to be specific), the growth spectrum not only depends on the ratio between the two principal stresses but also depends on the direction of the principal stress $\left(\theta_{p}\right)$. Even under an equi-biaxial stress, an anisotropic growth spectrum emerges, with four peaks aligned in the two orthogonal crystal directions, [100] and [010]. In this case, while the stress state is isotropic, the anisotropic elastic property of the crystal film breaks the rotational symmetry. As a result, an orthogonally oriented bi-directional pattern is predicted to dominate the initial growth of the wrinkles. By varying the ratio $\sigma_{2} / \sigma_{1}$ from 1 to 0 , the growth spectrum changes from orthogonal to uniaxial in the direction of the major principal stress. The transition however depends on the principal angle $\theta_{p}$, as shown in the second and third rows of Figure 4 for $\theta_{p}=0$ and $45^{\circ}$, respectively. For $\theta_{p}=0$, the growth spectrum remains orthogonal when the ratio $\sigma_{2} / \sigma_{1}$ slightly deviates from 1 , but the peak growth rate becomes lower in one 
direction compared to the other direction. The growth spectrum becomes uniaxial as the lower peak diminishes for $\sigma_{2} / \sigma_{1}<0.8$. For $\theta_{p}=45^{\circ}$, the wave vectors corresponding to the peak growth rates rotate toward the direction of the major principal stress and eventually merge into a uniaxial pattern. At an intermediate stress ratio (e.g., $\sigma_{2} / \sigma_{1}=0.9$ ), four peaks lie on two directions of an oblique angle. Hence, the kinetically dominant wrinkle pattern at the initial stage becomes obliquely oriented bi-directional (e.g, the zigzag pattern). The different transition in the initial wrinkle patterns can be understood as a result of the competition between the material anisotropy and the stress anisotropy through $\bar{E}_{\theta}$ and $\sigma_{\theta}$, respectively.

\section{Nonlinear Analysis of Equilibrium States}

For a viscoelastic substrate layer with a rubbery modulus $\mu_{R}>0$ at the long-time limit, wrinkling of an elastic film atop evolves towards an equilibrium state, dictated by minimization of the elastic strain energy stored in the film and the substrate. It is noted that there may exist many mechanically equilibrium states, including the one at the reference state with no wrinkles at all. However, by the principle of thermodynamics these equilibrium states may be unstable, stable, or metastable. Searching for the thermodynamically equilibrium state with the minimum energy requires consideration of all possible wrinkle patterns. In practice, several simple wrinkle patterns (e.g., parallel stripes, checkerboard, and herringbone) have been considered for isotropic elastic films (Chen and Hutchinson, 2004; Huang et al., 2005; Song et al., 2008). However, experiments have observed more complex wrinkle patterns (e.g., labyrinth). In the present study, facilitated by the kinetics of viscoelastic deformation in the substrate layer, we simulate the evolution of wrinkle patterns from a randomly generated initial perturbation. As the viscoelastic deformation dissipates energy, the evolution process may be regarded as a searching algorithm 
for the minimum energy state, but it is not guaranteed that the global minimum can be reached. Nevertheless, the viscoelastic evolution represents one physical process to form wrinkle patterns in experiments (e.g., Hobart et al., 2000; Yoo and Lee, 2003; Peterson et al., 2006), where the observed wrinkle patterns may also be trapped in a state of local energy minimum. It is suspected that there may exist many local minima in the energy landscape, and the observed wrinkle pattern may depend on the loading history (Biot, 1957).

This section presents a nonlinear energy analysis for parallel-striped winkles of a cubic crystal film on a thin viscoelastic substrate layer. The result provides useful insight into ordering of wrinkle patterns under various stress states and will be compared to the numerical simulations in the next section.

The elastic strain energy in the film consists of two parts, one associated with the in-plane deformation and the other with bending. Taking the strain energy at the reference state to be zero, the in-plane strain energy per unit area of the film is

$$
U_{C}=h\left(\sigma_{x x}^{R} \varepsilon_{x x}+\sigma_{y y}^{R} \varepsilon_{y y}+2 \sigma_{x y}^{R} \varepsilon_{x y}+\frac{1}{2} \sigma_{x x} \varepsilon_{x x}+\frac{1}{2} \sigma_{y y} \varepsilon_{y y}+\sigma_{x y} \varepsilon_{x y}\right) .
$$

By substituting Eqs. (5) and (6) into Eq. (24) and neglecting the in-plane displacements, we obtain that

$$
\begin{aligned}
U_{C}= & \frac{1}{2} h\left\{\sigma_{x x}^{R}\left(\frac{\partial w}{\partial x}\right)^{2}+\sigma_{y y}^{R}\left(\frac{\partial w}{\partial y}\right)^{2}+2 \sigma_{x y}^{R}\left(\frac{\partial w}{\partial x} \frac{\partial w}{\partial y}\right)\right\} \\
& +\frac{1}{8} h\left[\bar{C}_{11}\left(\frac{\partial w}{\partial x}\right)^{4}+\bar{C}_{22}\left(\frac{\partial w}{\partial y}\right)^{4}+2\left(\bar{C}_{12}+2 C_{66}\right)\left(\frac{\partial w}{\partial x} \frac{\partial w}{\partial y}\right)^{2}\right]
\end{aligned}
$$

The area density of the bending strain energy in a cubic crystal film is:

$$
U_{B}=\frac{h^{3}}{24}\left[\bar{C}_{11}\left(\frac{\partial^{2} w}{\partial x^{2}}\right)^{2}+\bar{C}_{22}\left(\frac{\partial^{2} w}{\partial y^{2}}\right)^{2}+2 \bar{C}_{12}\left(\frac{\partial^{2} w}{\partial x^{2}}\right)\left(\frac{\partial^{2} w}{\partial y^{2}}\right)+4 C_{66}\left(\frac{\partial^{2} w}{\partial x \partial y}\right)^{2}\right]
$$


At the equilibrium state, by setting $\partial w / \partial t=0$ in Eq. (10), the pressure acting on the substrate surface is:

$$
p=-\frac{2(1-v)}{1-2 v} \frac{\mu_{R}}{H} w .
$$

The reversible elastic strain energy stored in the substrate (per unit area of the surface) is then

$$
U_{S}=-\frac{1}{2} p w=\frac{1-v}{1-2 v} \frac{\mu_{R}}{H} w^{2}
$$

Now consider a parallel-striped wrinkle described by Eq. (13). By integrating the strain energy density, Eqs. (25), (26), and (28), over one wavelength of the wrinkle and dividing by the wavelength ( $\lambda=2 \pi / k)$, the average strain energy per unit area of the film is obtained as follows:

$$
\begin{aligned}
& \bar{U}_{C}=\frac{1}{4} \sigma_{\theta} h k^{2} A^{2}+\frac{3}{64} \tilde{E}_{\theta} h k^{4} A^{4}, \\
& \bar{U}_{B}=\frac{1}{48} \bar{E}_{\theta} h^{3} k^{4} A^{2}, \\
& \bar{U}_{S}=\frac{1-v}{2(1-2 v)} \frac{\mu_{R}}{H} A^{2},
\end{aligned}
$$

where

$$
\widetilde{E}_{\theta}=\bar{C}_{11} \sin ^{4} \theta+\bar{C}_{22} \cos ^{4} \theta+2\left(\bar{C}_{12}+2 C_{66}\right) \sin ^{2} \theta \cos ^{2} \theta
$$

Equation (32) defines another anisotropic parameter, $\widetilde{E}_{\theta}$, which is different from $\bar{E}_{\theta}$ in Eq. (15) for an orthotropic plate. In the case of a cubic crystal plate, $\widetilde{E}_{\theta} \equiv \bar{E}_{\theta}$.

The total strain energy (per unit area of the reference state) at the equilibrium state is therefore

$$
\bar{U}(A, k, \theta)=\bar{U}_{C}+\bar{U}_{B}+\bar{U}_{S}
$$


For an arbitrary wave vector, minimizing the total energy gives the equilibrium amplitude as a function of $k$ and $\theta$ :

$$
A_{e}(k, \theta)=\frac{2 \sqrt{6}}{3 k}\left[-\frac{\sigma_{\theta}}{\widetilde{E}_{\theta}}-\frac{\bar{E}_{\theta}}{12 \widetilde{E}_{\theta}}(k h)^{2}-\frac{2(1-v)}{1-2 v} \frac{\mu_{R}}{\widetilde{E}_{\theta}} \frac{1}{k^{2} H h}\right]^{1 / 2} .
$$

When $-\sigma_{\theta}<-\sigma_{\theta c}$, Eq. (34) yields real values of the wrinkle amplitude for certain wave vectors, and the total strain energy at the equilibrium state is negative, i.e., $\bar{U}_{e}(k, \theta)=\bar{U}\left(A_{e}, k, \theta\right)<0$. The spectra of the energy, $\bar{U}_{e}(k, \theta)$, are plotted in Figure 5 for both isotropic and anisotropic elastic films under various residual stress states. Similar to Figure 4, contours of the normalized strain energy, $\bar{U}_{e} /\left(C_{11} h\right)$, are plotted in the plane spanning the $x$ and $y$ components of the normalized wave vector; only negative energy values are plotted. The major principal stress is fixed to be $\sigma_{1} / C_{11}=-0.003$, while the ratio $\sigma_{2} / \sigma_{1}$ is varied from 1 to 0 .

For an isotropic elastic film, the energy contours are concentric circles under an equibiaxial residual stress $\left(\sigma_{2} / \sigma_{1}=1\right)$. The strain energy minimizes on a circle of a particular radius, with no favored direction due to rotational symmetry of the isotropic system. Once the ratio between the two principal stresses deviates from 1 , the rotational symmetry is broken and the energy spectrum has two minima symmetrically located on the axis parallel to the direction of the major principal stress. Hence, an energetically favored wrinkle pattern emerges with ordered parallel stripes perpendicular to the direction of the major principal stress. However, it should be noted that, since only parallel striped wrinkle patterns are considered in the present analysis, the energy minima in the spectra are not necessarily global minima. For example, it has been shown that herringbone and checkerboard wrinkle patterns may have lower energy than the parallel striped pattern under equi-biaxial stress states (Chen and Hutchinson, 2004; Huang et al., 2005; Song et al., 2008). 
For a cubic crystal film ( $\mathrm{Si}_{0.7} \mathrm{Ge}_{0.3}$, to be specific), the energy spectrum depends on both the material anisotropy and the stress anisotropy. Under an equi-biaxial stress, there exist four energy minima aligned in the two orthogonal crystal directions, [100] and [010]. Thus, an energetically favored equilibrium wrinkle pattern may consist of parallel stripes in two orthogonal directions. When the two principal stresses are different, the energy spectrum depends on the principal direction $\theta_{p}$. When $\theta_{p}=0$, two energy minima are symmetrically located on the axis parallel to the direction of the major principal stress, while the other two minima in the orthogonal direction first become shallower and then disappear. When $\theta_{p} \neq 0$, as the stress ratio decreases from 1 to 0 , the wave vectors of the energy minima first rotate toward the direction of the major principal stress and then merge to form two minima in the same direction. Therefore, different equilibrium wrinkle patterns may emerge between the orthogonal and the uniaxial patterns.

By minimizing the strain energy, $\bar{U}_{e}(k, \theta)$, with respect to $k$ for a fixed angle $\theta$, the equilibrium wavelength is obtained as a function of $\theta$ :

$$
\lambda_{e}(\theta)=\frac{2 \pi}{k_{e}(\theta)}=\pi h\left[\frac{2(1-2 v)}{3(1-v)} \frac{H}{h} \frac{\bar{E}_{\theta}}{\mu_{R}}\right]^{1 / 4} .
$$

Substitution of the equilibrium wavelength into Eq. (34) gives the equilibrium amplitude for the parallel wrinkles with the angle $\theta$. Further minimization of the strain energy, $\bar{U}_{e}\left(k_{e}, \theta\right)$, with respect to $\theta$ gives the angle for the parallel wrinkles with the minimum energy. Both the equilibrium wavelength and the angle can be determined from the locations of the minima in the energy spectrum shown in Figure 5. It should be noted that, although the energy spectra in Figure 5 appear similar to the initial growth spectra in Figure 4, the locations for the energy minima are different from those of the maximum growth rates. While the fastest growing wavelength (Eq. 
22) depends on the residual stress in the film, the equilibrium wrinkle wavelength (Eq. 35) is independent of the stress. Under the condition $-\sigma_{\theta}<-\sigma_{\theta c}$, the equilibrium wavelength is always greater than the fastest growing wavelength at the initial stage. Consequently, the wrinkle wavelength coarsens as the wrinkle evolves. Coarsening of wrinkle patterns of isotropic elastic films has been observed in both experiments (Yoo and Lee, 2003) and numerical simulations (Huang and Im, 2006).

Figure 6a shows that, for the SiGe crystal film, the equilibrium wavelength, $\lambda_{e}(\theta)$, maximizes at the $<110>$ directions $\left(\theta= \pm 45^{\circ}\right)$ and minimizes at the $<100>$ directions $(\theta=0$ and $90^{\circ}$ ). The difference between the equilibrium wavelengths is about $5 \%$. In Figure $6 \mathrm{~b}$, the maximum and minimum equilibrium wavelengths are shown to decrease as the rubbery modulus increases. Figure $7 \mathrm{a}$ plots the equilibrium wrinkle amplitude versus the angle under an equibiaxial stress ( $\sigma_{1}=\sigma_{2}=-0.465 \mathrm{GPa}$ ), with a maximum at the $<100>$ directions ( $\theta=0$ and

$\left.90^{\circ}\right)$ and minimum at the $<110>$ directions $\left(\theta= \pm 45^{\circ}\right)$. The difference between the maximum and minimum amplitudes is about $12 \%$. Figure $7 \mathrm{~b}$ shows that the equilibrium wrinkle amplitude increases with the magnitude of the stress. For a given stress level, the wrinkle amplitude decreases as the rubbery modulus of the substrate increases and becomes zero beyond a critical value. As a reference, for a $\mathrm{Si}_{0.7} \mathrm{Ge}_{0.3}$ film epitaxially grown from a Si substrate, the lattice mismatch is $1.2 \%$ and the equi-biaxial mismatch stress is $-2.02 \mathrm{GPa}$.

\section{Numerical Simulations}

A spectral method was developed to simulate evolution of wrinkle patterns by numerically integrating the nonlinear equations, Eqs. (10)-(12), similar to that in the previous study for wrinkling of isotropic films (Huang and Im, 2006). For the present study, a square 
computational cell of size $L=2000 h$ is discretized into a 128 by 128 grid, with periodic boundary conditions. A random perturbation of amplitude $0.01 \mathrm{~h}$ was introduced as the initial lateral deflection from the reference state. Figure 8 shows an evolution sequence of the simulated wrinkle pattern for a cubic crystal film ( $\mathrm{Si}_{0.7} \mathrm{Ge}_{0.3}$, to be specific) under an equi-biaxial compression $\left(\sigma_{1}=\sigma_{2}=-0.003 C_{11}\right)$. The lateral deflection, $w(x . y, t)$, is normalized by the film thickness $h$ and plotted as contours in the $x-y$ plane; the time is normalized by the scale $\tau=\eta / C_{11}$. The insets in Figure 8 show Fourier transforms of the corresponding wrinkle patterns, as contours in the Fourier space. For each wrinkle pattern, the root-mean-square (RMS) of the lateral deflection and the average wrinkle wavelength $(\bar{\lambda})$ are calculated as follows:

$$
\begin{aligned}
& \operatorname{RMS}(t)=\sqrt{\frac{\sum w(m, n, t)^{2}}{N^{2}}}, \\
& \bar{\lambda}(t)=\frac{2 \pi}{\bar{k}(t)}=2 \pi \sqrt{\frac{\sum|\hat{w}(m, n, t)|^{2}}{\sum|\hat{w}(m, n, t)|^{2} k(m, n)^{2}}},
\end{aligned}
$$

where $w(m, n, t)$ is the deflection of the grid point $(m, n)$ at time $t, \hat{w}(m, n, t)$ is the intensity of the Fourier transform, $k(m, n)$ is the wave number of the grid point $(m, n)$ in the Fourier space, and $N$ is the number of grid points along one side of the computational cell (i.e., $N=128$ for the present study).

The initial perturbation at $t=0$ is featureless (Fig. 8a), with a small roughness (RMS $=$ 0.0057). At $t=10^{5}$ (Fig. 8b), the perturbation amplitude (RMS) has grown significantly, and the Fourier transform takes a shape similar to the growth-rate spectrum shown in Figure 4 for the anisotropic film under an equi-biaxial stress. At this stage, many Fourier components are growing simultaneously in different directions, resulting in a rather disordered wrinkle pattern. The average wavelength ( $\bar{\lambda}=44.77$ ) is close to the fastest growing wavelength ( $\lambda_{\theta m}=43.23$ for 
$\theta=0$ ) as predicted by the linear analysis (Eq. 22). At $t=5 \times 10^{5}$ (Fig. 8c), the Fourier components with the fastest growth rate start to dominate, and the wrinkles become increasingly aligned in the two orthogonal directions, [100] and [010]. At $t=10^{6}$ (Fig. 8d), the wrinkle pattern exhibits a bi-phase domain structure, with parallel stripes locally ordered in one of the two orthogonal directions in each domain. Further evolution of the wrinkle pattern shows two coarsening processes. First, the wavelength of each individual wrinkle stripe increases. As a result, the average wavelength of the wrinkle over the entire area increases. This is consistent with the analytical solutions as the equilibrium wavelength for the parallel striped wrinkles ( $\left.\lambda_{e}=56.32\right)$ is greater than the wavelength of the fastest growing mode $\left(\lambda_{m}=43.23\right)$ at the early stage. Furthermore, the bi-phase domain structure of the wrinkle pattern evolves with coarsening of the domain size, as can be seen clearly from $t=10^{6}$ (Fig. 8d) to $t=10^{7}$ (Fig. 8e). Both the wrinkle wavelength and the domain size seem to saturate after a long time evolution (Fig. 8f). It is thus postulated that the viscoelastic evolution process seeks to minimize the total strain energy in the film and the substrate not only by selecting an equilibrium wavelength for individual wrinkle stripes but also by selecting a particular domain size. Similar coarsening processes have been observed for isotropic films (Yoo and Lee, 2003; Huang and Im, 2006). The present simulation of the wrinkle pattern evolution qualitatively agrees with the experiments by Peterson (2006) for a SiGe film on a glass layer at an elevated temperature, although quantitative comparisons are not possible due to uncertainties in the viscoelastic properties of the glass. The orthogonally ordered wrinkle pattern (Fig. 8f) is also comparable to the wavy structures observed in biaxially stressed silicon membranes on a PDMS substrate (Choi et al., 2007).

Figure 9 compares the wrinkle patterns for isotropic and anisotropic elastic films under various stress states. All patterns were obtained by numerical simulations of long time evolution 
up to $t=10^{8}$. The major principal stress is fixed as $\sigma_{1} / C_{11}=-0.003$, while the stress ratio $\sigma_{2} / \sigma_{1}$ varies from 1 to 0 . For the isotropic film, the wrinkle pattern changes from a disordered labyrinth pattern under an equi-biaxial stress $\left(\sigma_{2} / \sigma_{1}=1\right)$ to a parallel striped pattern under a uniaxial stress $\left(\sigma_{2} / \sigma_{1}=0\right)$, as predicted by the energy spectra shown in Figure 5 . The squareshaped computational cell seems to have an effect on the wrinkle orientation under the equibiaxial stress, favoring the horizontal and vertical directions. Despite the numerical artifact, the wrinkle pattern is largely disordered as compared to the orthogonal pattern of the anisotropic film under the same stress. Under a slightly anisotropic stress $\left(\sigma_{2} / \sigma_{1}=0.9\right)$, the wrinkle tends to order into parallel stripes, but not fully achieved. Although the energy spectrum shown in Figure 5 has two minima in the direction of the major principal stress, the energy landscape is rather spread out with a low driving force toward the minimum-energy state. As the degree of stress anisotropy increases, the driving force for ordering increases, and increasingly ordered wrinkle patterns form. Note the dislocation-type defects in the parallel striped wrinkle patterns for $\sigma_{2} / \sigma_{1}=0.7$ and 0.5 ; the density of the defects decreases during evolution. Similar defects were observed in experiments (Ohzono and Shimomura, 2004; Efimenko et al., 2005).

For the anisotropic film ( $\mathrm{Si}_{0.7} \mathrm{Ge}_{0.3}$, to be specific), wrinkle patterns are shown in Figure 9 for two different orientations of the principal stresses, $\theta_{p}=0$ and $45^{\circ}$. In both cases, the wrinkle pattern changes from orthogonal to uniaxial as the stress ratio varies, but the transitional patterns are different. When $\theta_{p}=0$, the orthogonal pattern remains orthogonal as the stress ratio slightly deviates from 1, but the bi-phase domain structure changes. The wrinkle pattern under the equibiaxial stress $\left(\sigma_{2} / \sigma_{1}=1\right)$ has parallel stripes in the two orthogonal directions, [100] and [010], each decorating about half of the area. For $\sigma_{2} / \sigma_{1}=0.9$, the area decorated with wrinkles in the 
[100] direction is greater than the area decorated by wrinkles in the [010] direction. The wrinkles in the [010] direction correspond to the local minima of the energy spectrum shown in Figure 5. As the stress ratio $\sigma_{2} / \sigma_{1}$ decreases, the area percentage of the [100] wrinkles increases, and the entire area is covered with the [100] wrinkles for $\sigma_{2} / \sigma_{1}<0.8$. Again, dislocation-type defects form during the evolution of parallel wrinkle patterns for $\sigma_{2} / \sigma_{1}=0.7$ and 0.5 . In the cases of $\theta_{p}=45^{\circ}$, the directions of the wrinkle stripes first rotate to form zigzag patterns $\left(\sigma_{2} / \sigma_{1}=0.9\right.$ and 0.8) and then merge into the [110] direction for $\sigma_{2} / \sigma_{1}<0.8$. The zigzag pattern consists of wrinkle stripes in two directions of an oblique angle, as predicted by the energy minima in the energy spectra (Fig. 5).

The effect of the stress magnitude on the wrinkle pattern is illustrated in Figure 10 for a crystal film under equi-biaxial stresses. As the stress magnitude increases, the wrinkle pattern becomes increasingly disordered. This is due to the fact that the stress state is isotropic and becomes dominant over the material anisotropy at the high stress levels. The contours of the initial growth rate show that the wavelength of the fastest growing mode decreases as the stress magnitude increases, while in the energy spectra the wavelengths of the energy minima remain constant. The average wavelengths of the wrinkle patterns $\left(t=10^{8}\right)$ are close to the predicted equilibrium value, $\lambda_{e}=56.32$, while the RMS roughness increases with the stress level. It is noted that both the growth rate and energy spectra become increasingly spread out as the stress magnitude increases, which may be responsible for the increasingly disordered wrinkle patterns. At a stress level very close to the critical stress, the growth and energy spectra become highly localized, leading to a well-ordered checkerboard pattern, as show in Figure 11. The wrinkle crests and troughs of the checkerboard pattern are organized orthogonally along the [100] and 
[010] directions of the cubic crystal. Experimentally, similar checkerboard patterns have been observed at the early stage of wrinkle evolution (Peterson, 2006), when the wrinkle amplitude is relatively small. Further growth of the wrinkle amplitude led to a transition to the orthogonal pattern with parallel stripes. Similar pattern transition is expected for the equilibrium state as the stress magnitude increases. Such a transition has its origin in a bifurcation of the local deformation of the elastic film, as the checkerboard pattern with largely spherical bending of the film gives way to a more energetically favorable stripe pattern with cylindrical bending of the film. Previously, Huang et al. (2005) showed that the checkerboard pattern may also form in isotropic elastic films, while the energy analysis (Chen and Hutchinson, 2004; Song et al., 2008) suggested that the checkerboard pattern is unfavorable compared to the zigzag pattern. No experimental observation of the checkerboard pattern has been reported for wrinkling of isotropic elastic films.

\section{Conclusions}

This paper presents a nonlinear model for wrinkling of an anisotropic crystal film on a viscoelastic substrate layer. A linear perturbation analysis is performed to predict onset of the wrinkling instability and the wrinkle evolution kinetics at the early stage, and an energy minimization approach is adopted to analyze the equilibrium wrinkle patterns. Numerical simulations are performed for a cubic crystal film under various stress states. A variety of wrinkle patterns (e.g., orthogonal, parallel, zigzag, and checkerboard patterns) emerge as a result of the competition between the material anisotropy and the stress anisotropy. Specifically, for wrinkling of cubic crystal SiGe films, the main conclusions are summarized as follows. 
- The critical stress for the onset of wrinkling depends on the wrinkle orientation, with the lowest critical stress in the $<100>$ directions and the highest in the $<110>$ directions.

- Under an equibiaxial residual stress, the wrinkle growth rate at the early stage maximizes in the $<100>$ directions, and the strain energy of parallel striped wrinkles at equilibrium states minimizes also in the $<100>$ directions; both suggest formation of orthogonally ordered wrinkle patterns.

- The equilibrium wrinkle wavelength and amplitude depends on the wrinkle orientation, with the minimum wavelength and maximum amplitude in the $<100>$ directions.

- Under an anisotropic stress, transition of the wrinkle pattern from orthogonal to parallel stripes depends on the angle of the principal stress, and zigzag patterns form in between.

- Formation of a checkerboard wrinkle pattern is shown by numerical simulations under an equi-biaxial stress close to the critical stress level. As the stress magnitude increases, the wrinkle pattern first changes to orthogonally ordered stripes and then becomes increasingly disordered.

\section{Acknowledgments}

The authors are grateful for the financial support by National Science Foundation under Grant No. 0547409. 


\section{References}

Allen, H.G., 1969. Analysis and Design of Structural Sandwich Panels, Pergamon, New York.

Biot, M.A., 1957. Folding instability of a layered viscoelastic medium under compression. Proc. R. Soc. A 242, 444-454.

Biot, M.A., 1963. Surface instability of rubber in compression. Applied Scientific Research A 12, 168-182.

Biot, M.A., 1965. Mechanics of Incremental Deformation. John Wiley \& Sons, Inc., New York.

Bloom, F., and Coffin, D., 2000. Handbook of Thin Plate Buckling and Postbuckling, Chapman \& Hall/CRC Press, New York.

Bowden, N., Brittain, S., Evans, A.G., Hutchinson, J.W., Whitesides, G.M., 1998. Spontaneous formation of ordered structures in thin films of metals supported on an elastomeric polymer. Nature 393, 146-149.

Chan, E.P., and Crosby, A.J., 2006. Fabricating microlens arrays by surface wrinkling. Advanced Materials 18, 3238-3242.

Chan, E.P., Smith, E.J., Hayward, R.C., Crosby, A.J., 2008. Surface wrinkles for smart adhesion. Advanced Materials 20, 711-716.

Chen, X., Hutchinson, J.W., 2004. Herringbone buckling patterns of compressed thin films on compliant substrates. J. Appl. Mech. 71, 597-603.

Choi, W.M., Song, J., Khang, D.-Y., Jiang, H., Huang, Y., Rogers, J.A., 2007. Biaxially stretchable “wavy” silicon nanomembranes. Nano Letters 7, 1655-1663.

Christensen, R.M., 1982. Theory of Viscoelasticity: An Introduction. Academic Press, New York.

Efimenko, K., Rackaitis, M., Manias, E., Vaziri, A., Mahadevan, L., Genzer, J., 2005. Nested self-similar wrinkling patterns in skins. Nature Materials 4, 293-297. 
Fitzgerald, E. A., 1995. GeSi/Si Nanostructures. Annu. Rev. Mater. Sci. 25, 417-454.

Genzer, J. and Groenewold, J., 2006. Soft matter with hard skin: from skin wrinkles to templating and material characterization. Soft Matter 2, 310-323.

Goodier, J.N., and Neou, I.M., 1951. The evaluation of theoretical critical compression in sandwich plates. J. Aero. Sci. 18, 649-657.

Gough, G.S., Elam, C.F., De Bruyne, N.D., 1940. The stabilization of a thin sheet by a continuous supporting medium. J. Roy Aero. Soc. 44, 12-43.

Groenewold, J., 2001. Wrinkling of plates coupled with soft elastic media. Physica A 298, 32-45.

Harrison, C., Stafford, C.M., Zhang, W., Karim, A., 2004. Sinusoidal phase grating created by a tunably buckled surface. Applied Physics Letters 85, 4016-4018.

Hobart, K.D., Kub, F.J., Fatemi, M., Twigg, M.E., Thompson, P.E., Kuan, T.S., Inoki, C.K., 2000. Compliant substrates: a comparative study of the relaxation mechanisms of strained films bonded to high and low viscosity oxides. J. Electron. Mater. 29, 897-900.

Huang, R., 2005. Kinetic wrinkling of an elastic film on a viscoelastic substrate. Journal of the Mechanics and Physics of Solids 53, 63-89.

Huang, R., Im, S.H., 2006. Dynamics of wrinkle growth and coarsening in stressed thin films. Physical Review E 74, 26214.

Huang, R., Suo, Z., 2002a. Wrinkling of a compressed elastic film on a viscous layer. Journal of Applied Physics 91, 1135-1142.

Huang, R., and Suo, Z., 2002b. Instability of a compressed elastic film on a viscous layer. Int. J. Solids and Structures 39, 1791-1802.

Huang, Z.Y., Hong, W., Suo, Z., 2005. Nonlinear analyses of wrinkles in a film bonded to a compliant substrate. Journal of the Mechanics \& Physics of Solids 53, 2101. 
Huck, W.T.S., Bowden, N., Onck, P., Pardoen, T., Hutchinson, J.W., Whitesides, G.M., 2000. Ordering of spontaneously formed buckles on planar surfaces. Langmuir 16, 3497-3501.

Im, S.H., and Huang, R., 2005. Evolution of wrinkles in elastic-viscoelastic bilayer thin films. J. Applied Mechanics 72, 955-961.

Jiang, H.Q., Khang, D.-Y., Song, J., Sun, Y., Huang, Y., Rogers, J.A., 2007. Finite deformation mechanics in buckled thin films on compliant supports. Proc. National. Acad. Sci. 104, 15607-15612.

Lacour, S.P., Wagner, S., Huang, Z.Y., Suo, Z., 2003. Stretchable gold conductors on elastomeric substrates. Appl. Phys. Lett. 82 (15), 2404-2406.

Landau, L.D., Lifshitz, E.M., 1959. Theory of Elasticity, Pergamon, London.

Lu, W., and Suo, Z., 2002. Symmetry breaking in self-assembled monolayers on solid surfaces. I. Anisotropic surface stress. Phys. Rev. B, 65, 085401.

Martin, S.J., Godschalx, J.P., Mills, M.E., Shaffer, E.O., Townsend, P.H., 2000. Development of a low-dielectric-constant polymer for the fabrication of integrated circuit interconnect. Advanced Materials 12, 1769-1778.

Matthews, J.W., and Blakeslee, A.E., 1974. Defects in epitaxial multilayers I. Misfit dislocations. J. Crystal Growth 27, 118-125.

Mumm, D.R., Evans, A.G., Spitsberg, I.T., 2001. Characterization of a cyclic displacement instability for a thermally grown oxide in a thermal barrier system. Acta Mater. 49, 23292340.

Ohzono, T., Shimomura, M., 2004. Ordering of microwrinkle patterns by compressive strain. Physical Review B 69, 132202. 
Pang, Y., and Huang, R., 2007. Bifurcation of surface pattern in epitaxial thin films under anisotropic stresses. J. Applied Physics 101, 023519.

Peterson, R.L., 2006. Stretching Silicon: A Uniaxial and Biaxial Strain Generation Process and the Resulting Mobility Enhancement in Silicon-on-Insulator MOSFETs. PhD dissertation, Princeton University.

Peterson, R.L., Hobart, K.D., Kub, F.J., Yin, H., Sturm, J.C., 2006. Reduced buckling in one dimension versus two dimensions of a compressively strained film on a compliant substrate. Applied Physics Letters 88, 201913.

Serrano, J.R., Cahill, D.G., 2002. Micron-scale buckling of $\mathrm{SiO}_{2}$ on Si. J. Appl. Phys. 92 (12), 7606-7610.

Song, J., Jiang, H., Choi, W.M., Khang, D.Y., Huang, Y., Rogers, J.A., 2008. An analytical study of two-dimensional buckling of thin films on compliant substrates. Journal of Applied Physics 103, 014303.

Sridhar, N., Srolovitz, D.J., Suo, Z., 2001. Kinetics of buckling of a compressed film on a viscous substrate. Applied Physics Letters 78, 2482-2484.

Stafford, C.M., Harrison, C., Beers, K.L., Karim, A., Amis, E.J., Vanlandingham, M.R., Kim, H.-C., Volksen, W., Miller, R.D., Simonyi, E.E., 2004. A buckling-based metrology for measuring the elastic moduli of polymeric thin films. Nature Materials 3, 545-550.

Suo, Z., 1995. Wrinkling of the oxide scale on an aluminum-containing alloy at high temperatures. J. Mech. Phys. Solids 43, 829-846.

Timoshenko, S., Woinowsky-Krieger, S., 1987. Theory of Plates and Shells (2 ${ }^{\text {nd }}$ ed.), McGrawHill, New York. 
Tolpygo, V.K., Clarke, D.R., 1998. Wrinkling of $\alpha$-aluminum films grown by thermal oxidationI: Quantitative studies on single crystals of Fe-Cr-Al alloy. Acta Mater. 46 (14), 5153-5166.

Volynskii, A.L., Bazhenov, S., Lebedeva, O.V., Bakeev, N.F., 2000. Mechanical buckling instability of thin coatings deposited on soft polymer substrates. J. Materials Science 35, 547554.

Wan, C.C., 1947. Face buckling and core strength requirements in sandwich construction. J. Aero. Sci. 14, 531-539.

Watanabe, M., Shirai, H., Hirai, T., 2002. Wrinkled polypyrrole electrode for electroactive polymer actuators. J. Appl. Phys. 92 (8), 4631-4637.

Yin, H., Huang, R., Hobart, K.D., Liang, J., Suo, Z., Shieh, S.R., Duffy, T.S., Kub, F.J., Sturm, J.C., 2003. Buckling suppression of SiGe islands on compliant substrates. Journal of Applied Physics 94, 6875-6882.

Yoo, P.J., Lee, H.H., 2003. Evolution of a stress-driven pattern in thin bilayer films: spinodal wrinkling. Physical Review Letters 91 (15), 154502.

Yu C.-Y., Chen, P.-W., Jan, S.-R., Liao, M.-H., Liao, K.-F., Liu, C.W., 2005. Buckled SiGe layers by the oxidation of $\mathrm{SiGe}$ on viscous $\mathrm{SiO}_{2}$ layers. Applied Physics Letters 86, 011909. 
Table 1. Elastic constants of single-crystal silicon (Si), germanium (Ge), and an alloy $\mathrm{Si}_{0.7} \mathrm{Ge}_{0.3}$. Also listed are the constants of an artificial isotropic material for comparison.

\begin{tabular}{|c|c|c|c|c|}
\hline Crystal & $C_{11}(\mathrm{GPa})$ & $C_{12}(\mathrm{GPa})$ & $C_{66}(\mathrm{GPa})$ & $\begin{array}{c}\text { Degree of } \\
\text { anisotropy, } \xi\end{array}$ \\
\hline $\mathrm{Si}$ & 166.2 & 64.4 & 79.8 & 1.57 \\
\hline $\mathrm{Ge}$ & 128.4 & 48.2 & 66.7 & 1.66 \\
\hline $\mathrm{Si}_{0.7} \mathrm{Ge}_{0.3}$ & 154.9 & 59.5 & 75.9 & 1.59 \\
\hline Isotropic & 161.7 & 69.3 & 46.2 & 1.00 \\
\hline
\end{tabular}




\section{List of Figures}

Figure 1. Schematic illustration of an elastic film on a viscoelastic substrate: (a) At the reference state, the film is flat and subject to a uniform residual stress; The coordinates for a cubic crystal film are shown. (b) At a wrinkled state, the film and the substrate deform concomitantly.

Figure 2. Residual stress of the film represented by stress elements in different orientations: (a) in the $x-y$ coordinates; (b) in the principal directions; (c) in an arbitrarily rotated direction.

Figure 3. (a) Critical stress for wrinkling versus the angle of the wrinkle wave vector for $\mathrm{Si}$, Ge, and SiGe films; (b) The maximum and minimum critical stresses for a SiGe film versus the rubbery modulus of the substrate layer. $(H / h=10, v=0.45)$

Figure 4. Spectra of the wrinkle growth rate at the early stage for isotropic and anisotropic elastic films under various residual stress states $\left(-0.2 \leq k_{x} h, k_{y} h \leq 0.2\right)$. The major principal stress is $\sigma_{1} / C_{11}=-0.003 ; H / h=10, v=0.45$, and $\mu_{R} / C_{11}=10^{-5}$.

Figure 5. Energy spectra of parallel wrinkles at the equilibrium state for isotropic and anisotropic elastic films under various residual stress states $\left(-0.2 \leq k_{x} h, k_{y} h \leq 0.2\right)$. The major principal stress is $\sigma_{1} / C_{11}=-0.003 ; H / h=10, v=0.45$, and $\mu_{R} / C_{11}=10^{-5}$.

Figure 6. (a) Equilibrium wavelength of parallel wrinkles as a function of the angle of the wrinkle wave vector for Si, Ge, and SiGe films; (b) The maximum and minimum equilibrium wavelengths for a SiGe film versus the rubbery modulus of the substrate layer. $(H / h=10$, $v=0.45$ ) 
Figure 7. (a) Equilibrium amplitude of parallel wrinkles as a function of the angle of the wrinkle wave vector for $\mathrm{Si}, \mathrm{Ge}$, and SiGe films under an equi-biaxial stress; (b) The maximum and minimum equilibrium wavelengths for a SiGe film versus the rubbery modulus of the substrate layer. $(H / h=10, v=0.45)$

Figure 8. A simulated evolution sequence of the wrinkle pattern for a $\mathrm{Si}_{0.7} \mathrm{Ge}_{0.3}$ film under an equi-biaxial stress $\left(\sigma_{1}=\sigma_{2}=-0.003 C_{11}, \mu_{R} / C_{11}=10^{-5}, H / h=10, v=0.45\right)$ : (a) $t=0$, $\mathrm{RMS}=0.0057, \bar{\lambda}=38.83$; (b) $t=10^{5}$, RMS $=0.0165, \bar{\lambda}=44.77$; (c) $t=5 \times 10^{5}$, RMS $=$ 0.4185, $\bar{\lambda}=47.06$; (d) $t=10^{6}$, RMS $=0.4676, \bar{\lambda}=50.75$; (e) $t=10^{7}$, RMS $=0.5876, \bar{\lambda}=$ 56.43; (f) $t=10^{8}$, RMS $=0.5918, \bar{\lambda}=56.63$.

Figure 9. Simulated wrinkle patterns $\left(t=10^{8}\right)$ for isotropic and anisotropic films under various residual stresses. The major principal stress is $\sigma_{1} / C_{11}=-0.003 ; \mu_{R} / C_{11}=10^{-5}, H / h=10$, $v=0.45$.

Figure 10. Contours of the initial growth rate and the energy at the equilibrium states $\left(-0.8 \leq k_{x} h, k_{y} h \leq 0.8\right)$, simulated wrinkle patterns $\left(t=10^{8}\right)$ for a cubic crystal film $\left(\mathrm{Si}_{0.7} \mathrm{Ge}_{0.3}\right)$ under equi-biaxial stresses with various stress magnitudes $\left(\mu_{R} / C_{11}=10^{-5}\right.$, $H / h=10, v=0.45)$.

Figure 11. (a) Energy spectrum of the equilibrium states and (b) the simulated wrinkle pattern ( $t$ $=10^{8}$ ) for a cubic crystal film $\left(\mathrm{Si}_{0.7} \mathrm{Ge}_{0.3}\right)$ under equi-biaxial stresses with the stress magnitude, $\sigma_{1}=\sigma_{2}=-0.00178 C_{11}$, close to the critical stress $\left(\sigma_{c}=-0.00177 C_{11}\right)$. RMS $=$ 0.05286 and $\bar{\lambda}=55.56$. (c) shows a magnified 3D view of the surface wrinkle. 


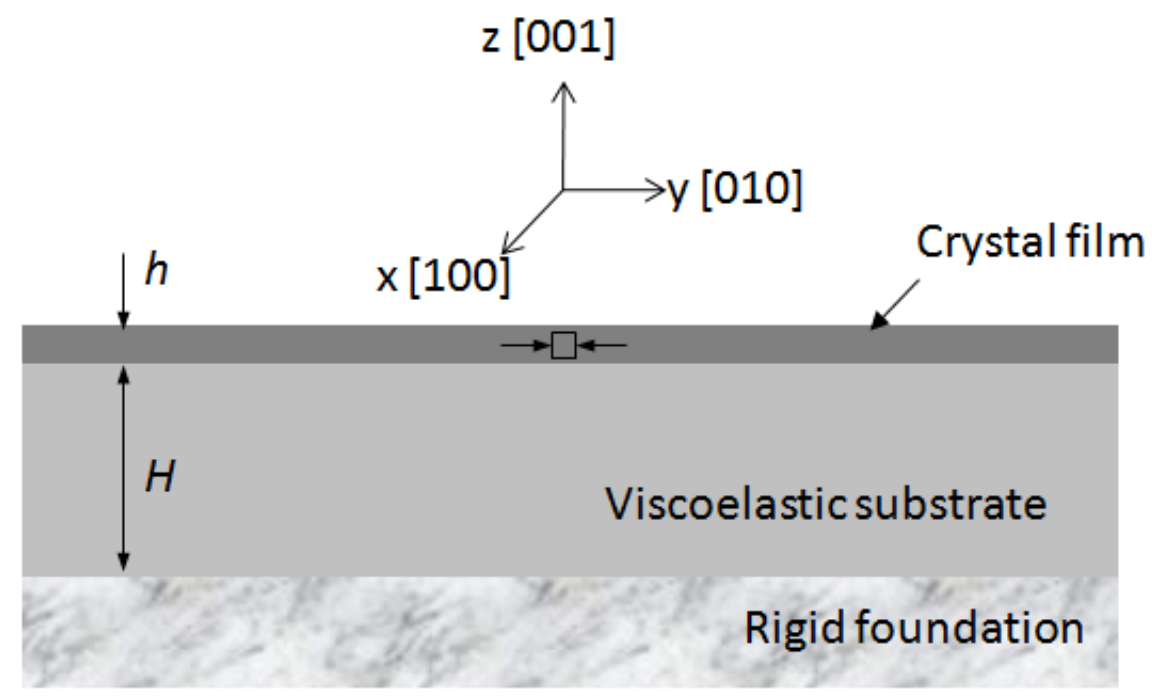

(a)

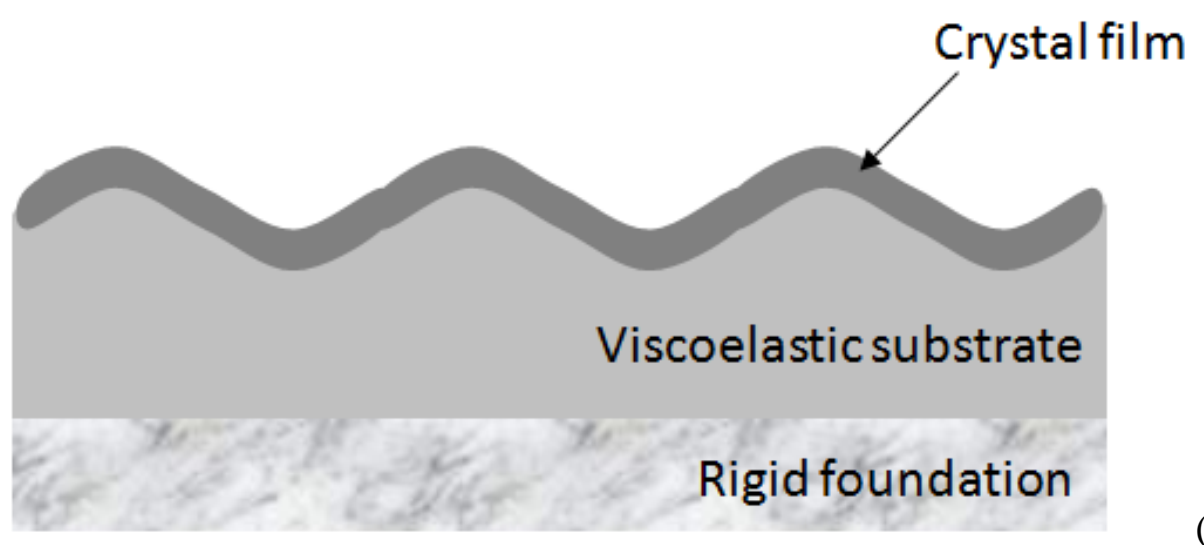

(b)

Figure 1. Schematic illustration of an elastic film on a viscoelastic substrate: (a) At the reference state, the film is flat and subject to a uniform residual stress; The coordinates for a cubic crystal film are shown. (b) At a wrinkled state, the film and the substrate deform concomitantly. 


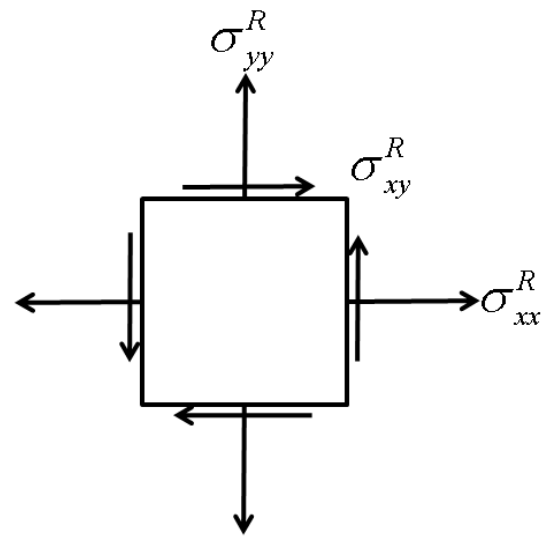

(a)

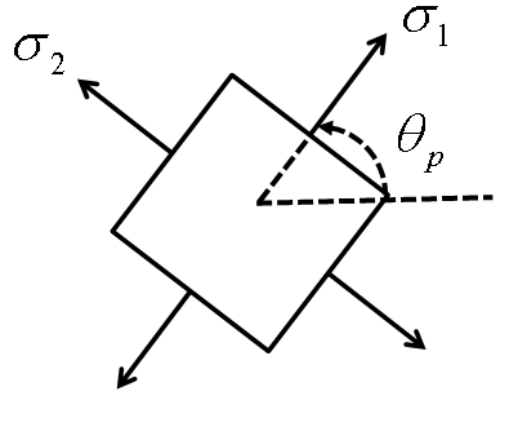

(b)

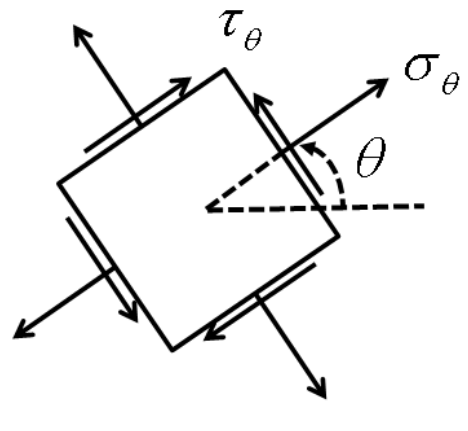

(c)

Figure 2. Residual stress of the film represented by stress elements in different orientations: (a) in the $x$-y coordinates; (b) in the principal directions; (c) in an arbitrarily rotated direction. 

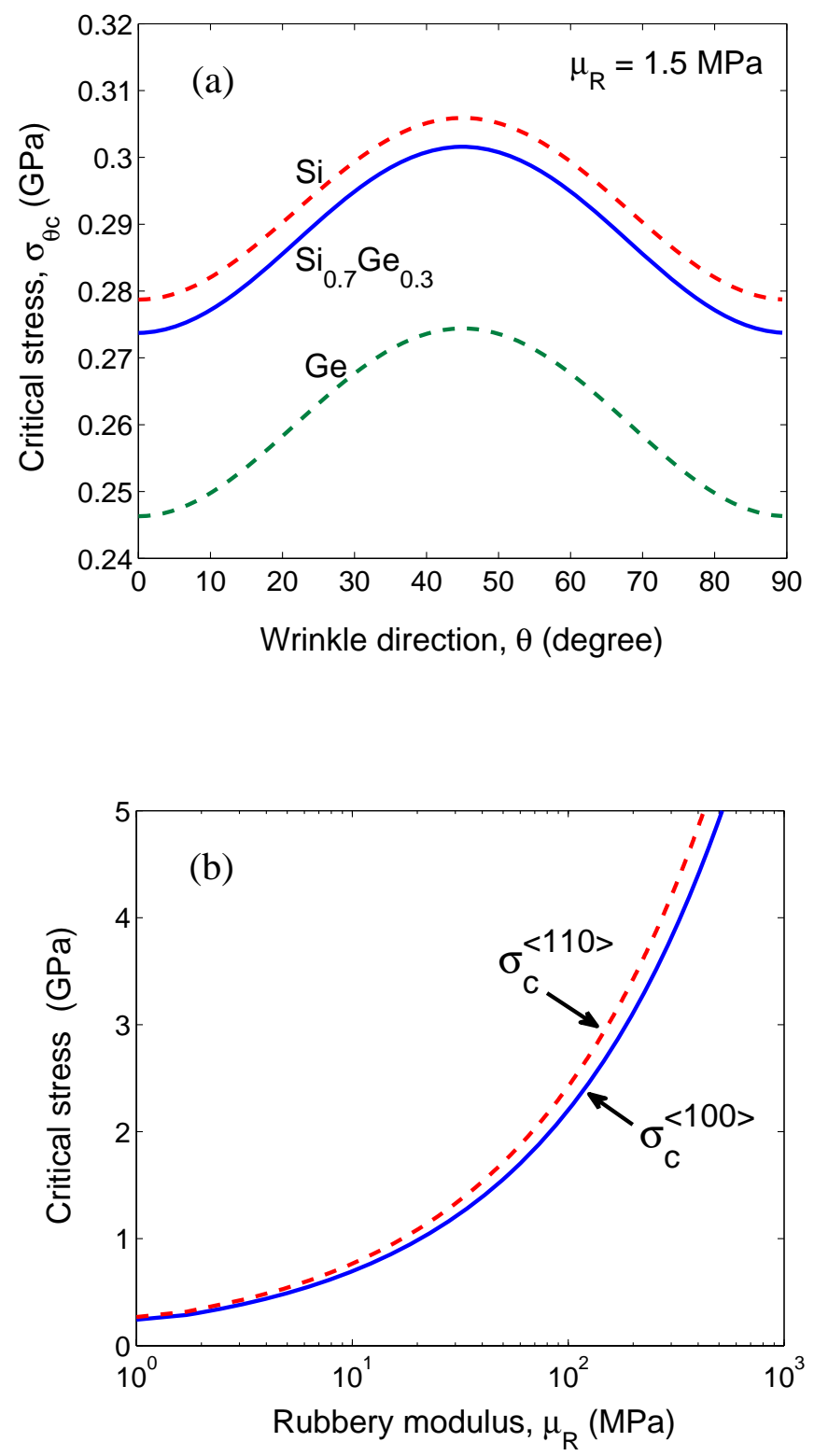

Figure 3. (a) Critical stress for wrinkling versus the angle of the wrinkle wave vector for $\mathrm{Si}, \mathrm{Ge}$, and SiGe films; (b) The maximum and minimum critical stresses for a SiGe film versus the rubbery modulus of the substrate layer. $(H / h=10, v=0.45)$ 


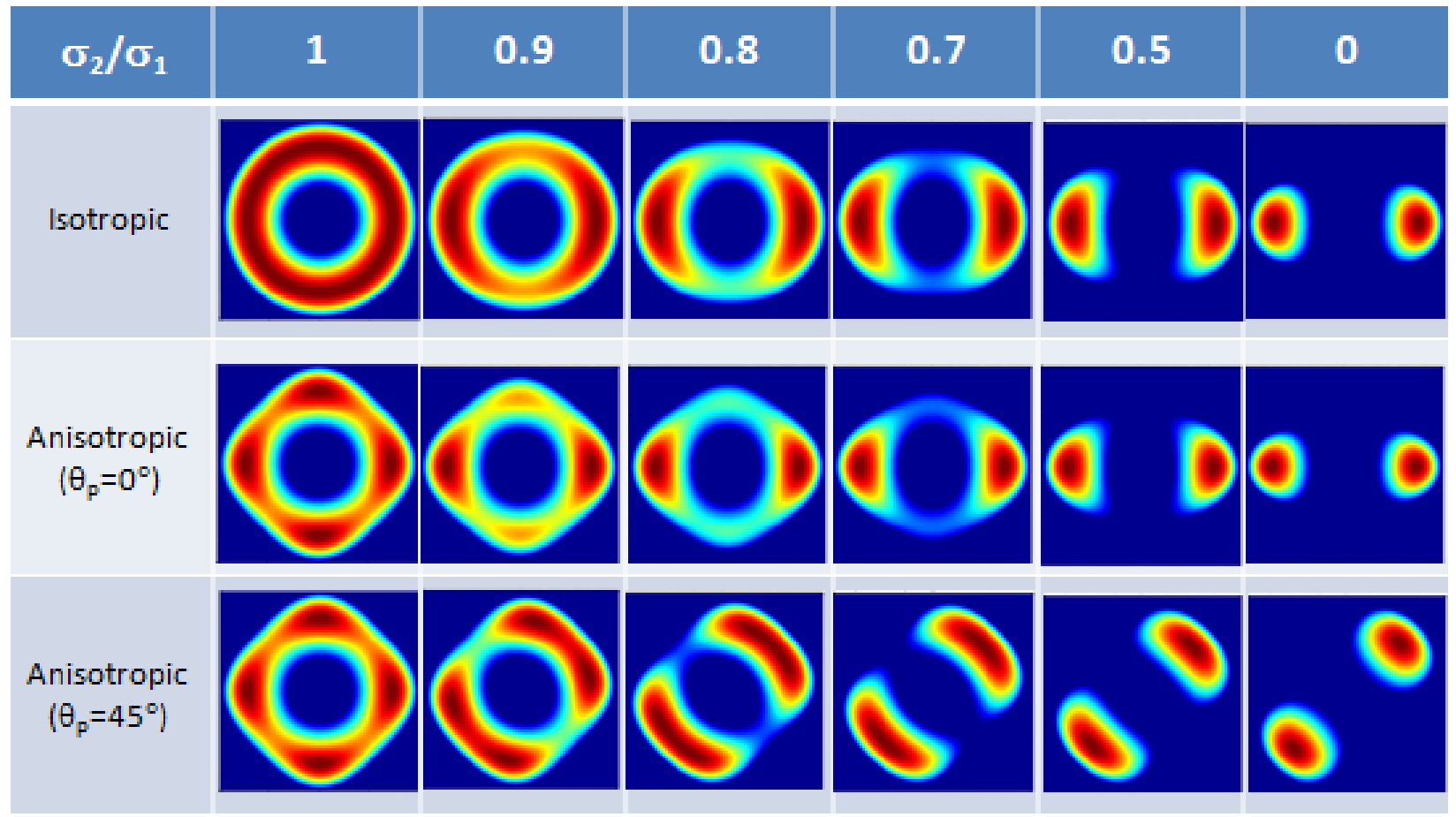

Figure 4. Spectra of the wrinkle growth rate at the early stage for isotropic and anisotropic elastic films under various residual stress states $\left(-0.2 \leq k_{x} h, k_{y} h \leq 0.2\right)$. The major principal stress is $\sigma_{1} / C_{11}=-0.003 ; H / h=10, v=0.45$, and $\mu_{R} / C_{11}=10^{-5}$. 


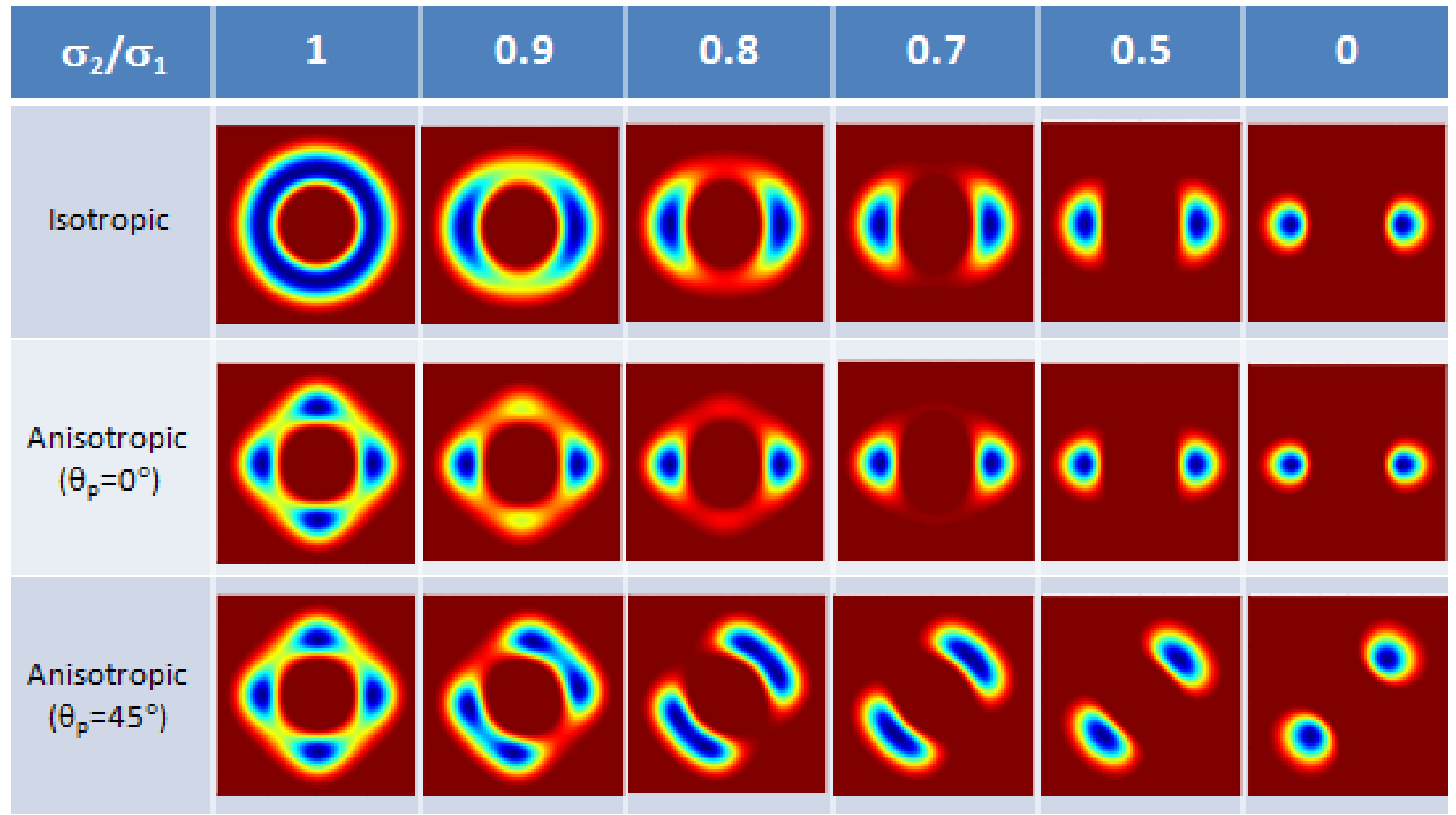

Figure 5. Energy spectra of parallel wrinkles at the equilibrium state for isotropic and anisotropic elastic films under various residual stress states $\left(-0.2 \leq k_{x} h, k_{y} h \leq 0.2\right)$. The major principal stress is $\sigma_{1} / C_{11}=-0.003 ; H / h=10, v=0.45$, and $\mu_{R} / C_{11}=10^{-5}$. 

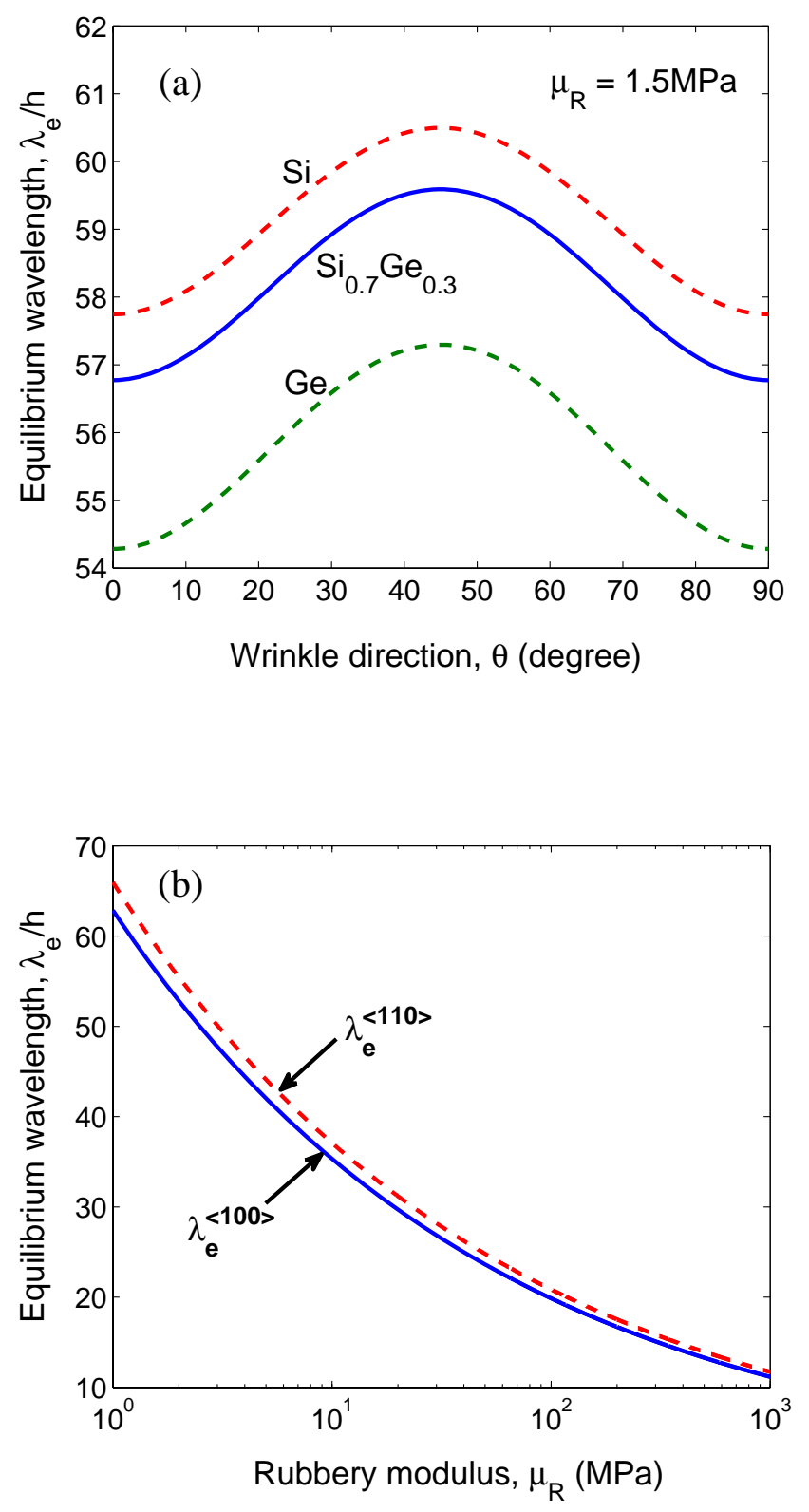

Figure 6. (a) Equilibrium wavelength of parallel wrinkles as a function of the angle of the wrinkle wave vector for Si, Ge, and SiGe films; (b) The maximum and minimum equilibrium wavelengths for a SiGe film versus the rubbery modulus of the substrate layer. ( $H / h=10$, $v=0.45)$ 

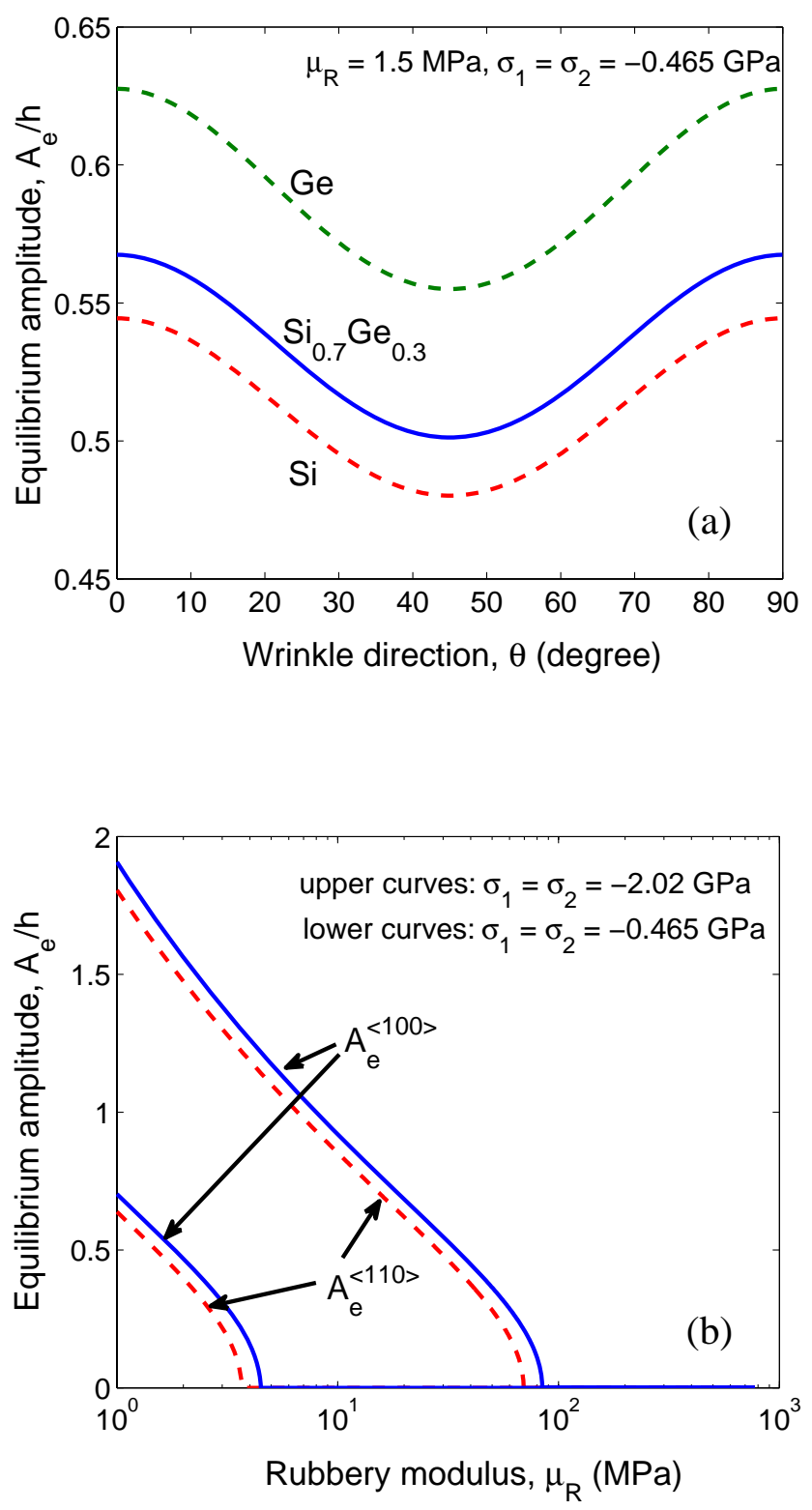

Figure 7. (a) Equilibrium amplitude of parallel wrinkles as a function of the angle of the wrinkle wave vector for $\mathrm{Si}, \mathrm{Ge}$, and SiGe films under an equi-biaxial stress; (b) The maximum and minimum equilibrium wavelengths for a SiGe film versus the rubbery modulus of the substrate layer. $(H / h=10, v=0.45)$ 

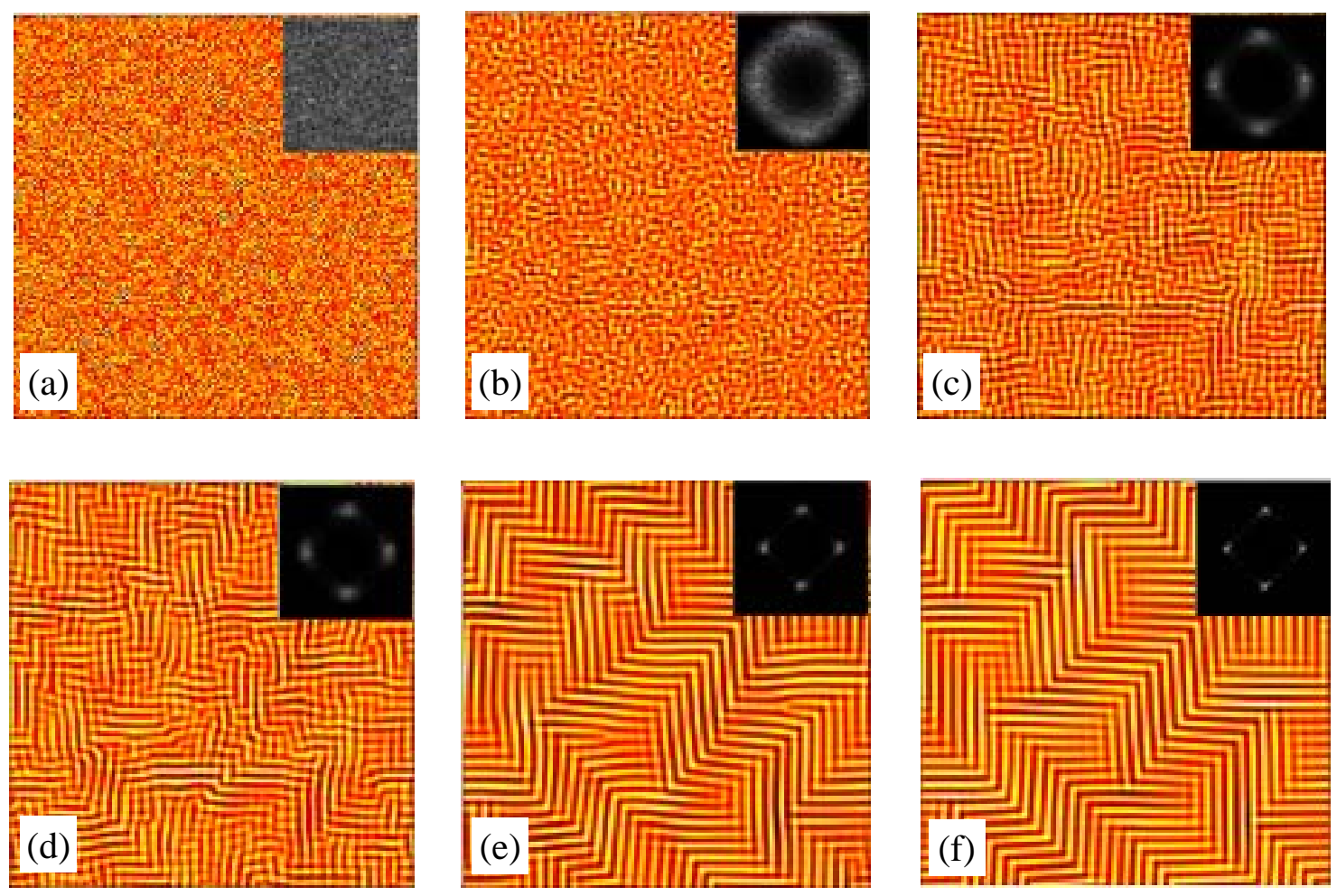

Figure 8. A simulated evolution sequence of the wrinkle pattern for a $\mathrm{Si}_{0.7} \mathrm{Ge}_{0.3}$ film under an equi-biaxial stress $\left(\sigma_{1}=\sigma_{2}=-0.003 C_{11}, \mu_{R} / C_{11}=10^{-5}, H / h=10, v=0.45\right)$ : (a) $t=0$, RMS $=$ 0.0057, $\bar{\lambda}=38.83$; (b) $t=10^{5}$, RMS $=0.0165, \bar{\lambda}=44.77$; (c) $t=5 \times 10^{5}$, RMS $=0.4185, \bar{\lambda}=$ 47.06; (d) $t=10^{6}$, RMS $=0.4676, \bar{\lambda}=50.75$; (e) $t=10^{7}$, RMS $=0.5876, \bar{\lambda}=56.43$; (f) $t=10^{8}$, RMS $=0.5918, \bar{\lambda}=56.63$. 


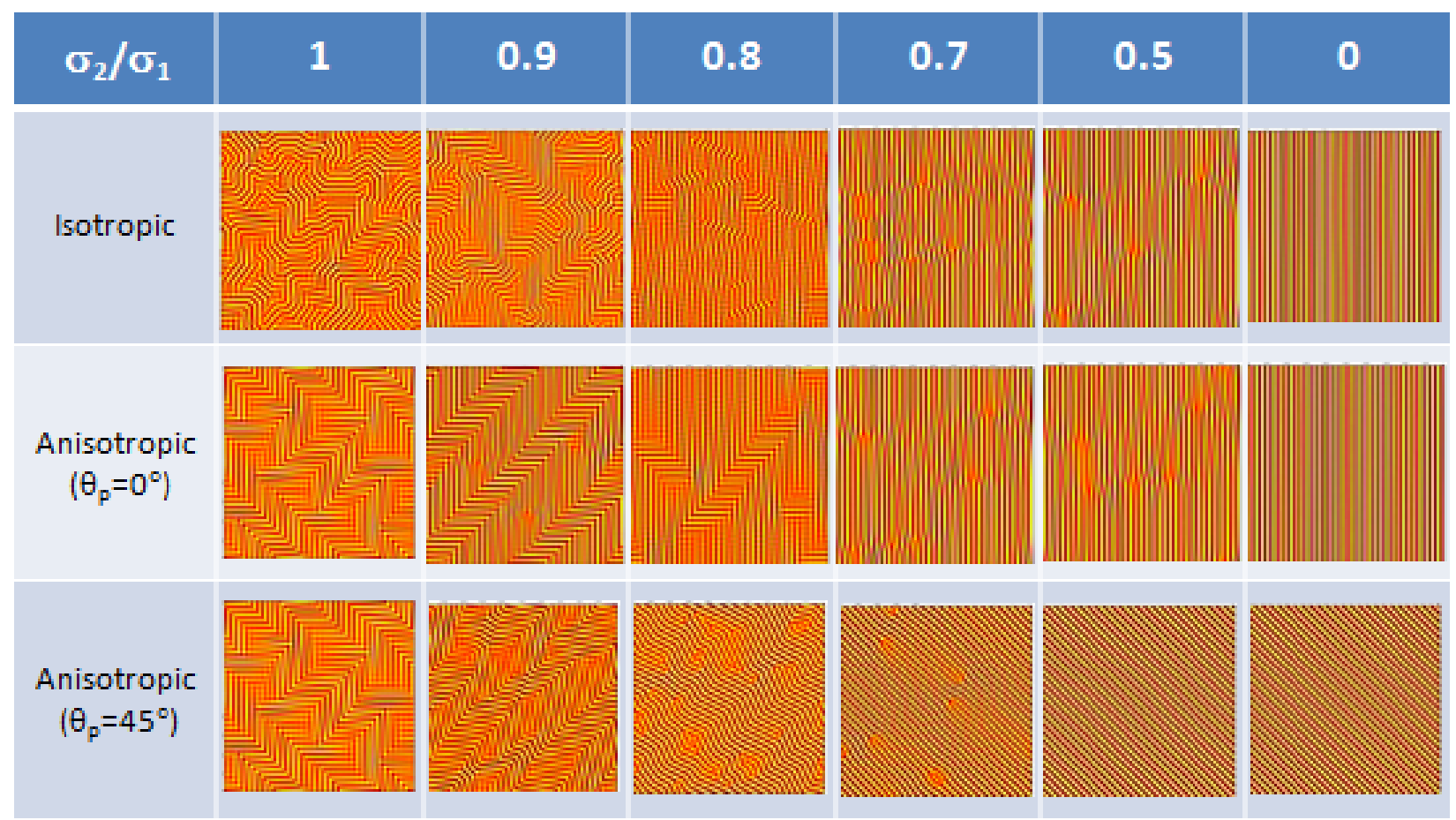

Figure 9. Simulated wrinkle patterns $\left(t=10^{8}\right)$ for isotropic and anisotropic films under various residual stresses. The major principal stress is $\sigma_{1} / C_{11}=-0.003 ; \mu_{R} / C_{11}=10^{-5}, H / h=10$, $v=0.45$. 


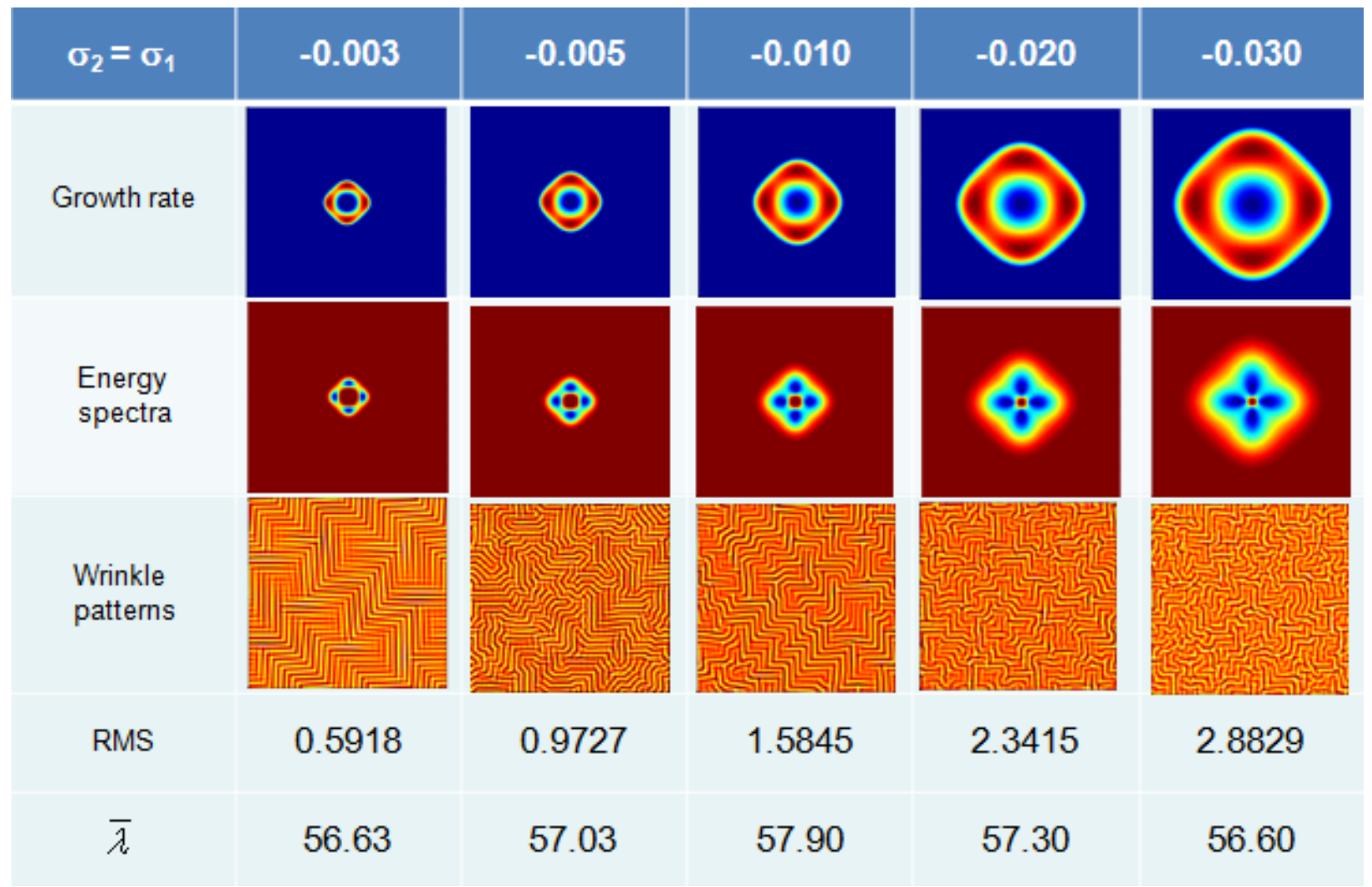

Figure 10. Contours of the initial growth rate and the energy at the equilibrium states $\left(-0.8 \leq k_{x} h, k_{y} h \leq 0.8\right)$, simulated wrinkle patterns $\left(t=10^{8}\right)$ for a cubic crystal film $\left(\mathrm{Si}_{0.7} \mathrm{Ge}_{0.3}\right)$ under equi-biaxial stresses with various stress magnitudes $\left(\mu_{R} / C_{11}=10^{-5}, H / h=10, v=0.45\right)$. 

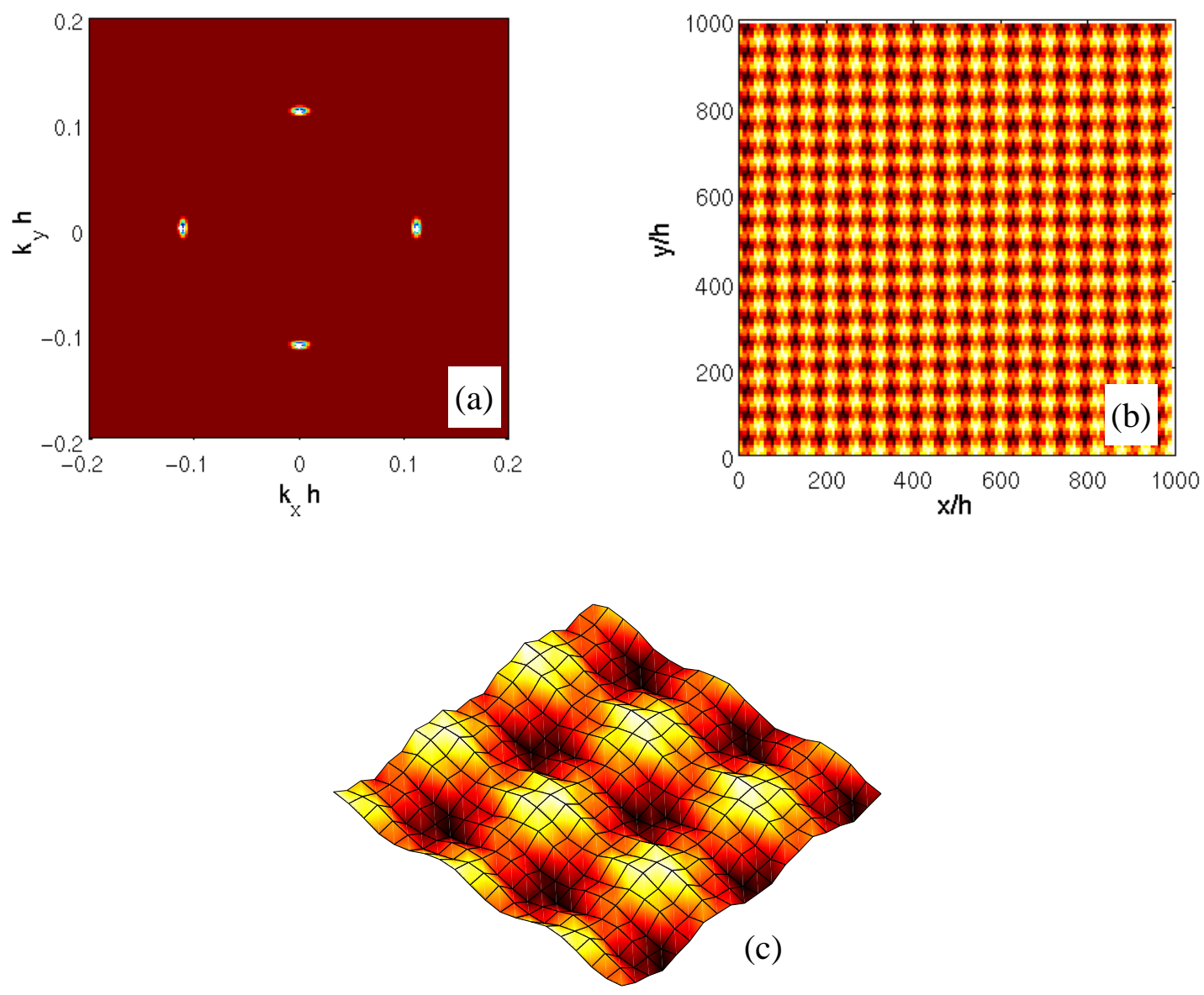

Figure 11. (a) Energy spectrum of the equilibrium states and (b) the simulated wrinkle pattern ( $t$ $=10^{8}$ ) for a cubic crystal film $\left(\mathrm{Si}_{0.7} \mathrm{Ge}_{0.3}\right)$ under equi-biaxial stresses with the stress magnitude, $\sigma_{1}=\sigma_{2}=-0.00178 C_{11}$, close to the critical stress $\left(\sigma_{c}=-0.00177 C_{11}\right)$. RMS $=0.05286$ and $\bar{\lambda}=55.56$. (c) shows a magnified 3D view of the surface wrinkle. 